\title{
A Human-Robot Cooperative and Personalized Compliant Joint Controller for Upper-Limb Rehabilitation Robots: The Elbow Joint Validation
}

Stefano Dalla Gasperina ( $\nabla$ stefano.dallagasperina@polimi.it)

Politecnico di Milano https://orcid.org/0000-0002-3466-8397

Valeria Longatelli

Politecnico di Milano

Francesco Braghin

Politecnico di Milano

Alessandra Laura Giulia Pedrocchi

Politecnico di Milano

Marta Gandolla

Politecnico di Milano

\section{Research}

Keywords: Compliant control, cooperative control, collaborative robotics, impedance, physical 28 humanrobot interaction, post-stroke, rehabilitation, upper limb, EMG

Posted Date: June 18th, 2021

DOI: https://doi.org/10.21203/rs.3.rs-595139/v1

License: (c) (i) This work is licensed under a Creative Commons Attribution 4.0 International License.

Read Full License 
- A human-robot cooperative and personalized compliant joint . controller for upper-limb rehabilitation robots: the elbow joint

\author{
validation. \\ Stefano Dalla Gasperina ${ }^{1}$, Valeria Longatelli ${ }^{1}$, Francesco Braghin ${ }^{2}$, \\ Alessandra Laura Giulia Pedrocchi ${ }^{1}$, and Marta Gandolla ${ }^{2}$
}

June 9, 2021

\begin{abstract}
Background: Appropriate training modalities for post-stroke upper-limb rehabilitation are key features for effective recovery after the acute event. This work presents a novel human-robot cooperative control framework that promotes compliant motion and renders different high-level human-robot interaction rehabilitation modalities under a unified low-level control scheme.

Methods: The presented control law is based on a loadcell-based impedance controller provided with positive-feedback compensation terms for disturbances rejection and dynamics compensation. We developed an elbow flexion-extension experimental setup, and we conducted experiments to evaluate the controller performances. Seven high-level modalities, characterized by different levels of (i) impedance-based corrective assistance, (ii) weight counterbalance assistance, and (iii) resistance, have been defined and tested with 14 healthy volunteers.

Results: The unified controller demonstrated suitability to promote good transparency and render compliant and high-impedance behavior at the joint. Superficial electromyography results showed different muscular activation patterns according to the rehabilitation modalities. Results suggested to avoid weight counterbalance assistance, since it could induce different motor relearning with respect to purely impedance-based corrective strategies.

Conclusion: We proved that the proposed control framework could implement different physical human-robot interaction modalities and promote the assist-as-needed paradigm, helping the user to accomplish the task, while maintaining physiological muscular activation patterns. Future insights involve the extension to multiple degrees of freedom robots and the investigation of an adaptation control law that makes the controller learn and adapt in a therapist-like manner.
\end{abstract}

Keywords - Compliant control, cooperative control, collaborative robotics, impedance, physical human-robot interaction, post-stroke, rehabilitation, upper limb, EMG.

\title{
1 Introduction
}

Worldwide, stroke is a leading cause of death and permanent disability [31]. Although the global mortality of stroke has decreased in the past decades, the incidence and the effects of the disease are expected to increase [22]. Consequently, the burden of stroke is still likely to produce long-term 
impairment, limitations during activities of daily living, and compromise the social participation of most stroke survivors. In most cases, rehabilitation treatment is required for an effective recovery, besides partial spontaneous recovery. Indeed, physical therapy fosters the motor relearning process during post-stroke rehabilitation. Nevertheless, only $5 \%$ to $20 \%$ of people with initial upper limb impairment after stroke completely recover lost functionalities [19]. In the past years, the literature proposed upper limb robot-assisted rehabilitation as a method to stimulate motor relearning through repetitive, high-intensity, task-oriented functional training [17,68]. Since the 90s, several upperlimb robotic devices have been designed, but only a few of them effectively reached the market, probably due to controversial results obtained in clinical trials [2].

Recent systematic reviews show that robotic rehabilitation could produce better, or at least equivalent, outcomes than conventional therapy in both the International Classification of Functioning, Disability and Health (ICF) Body and Activity domains [49,50,67]. Moreover, given that traditional passive mobilization limits neuroplasticity, a more customizable and adaptable control approach, facilitating subject's engagement and motivation, could lead to better effectiveness of the treatment [45]. Thus, the effectiveness of the robotic rehabilitation therapy strongly depends on the capability of the system to guide natural coordinated motion, promote physiological muscular contraction, and induce the patient to cooperate as much as possible. This is why a key component of effective robot-mediated therapy is a good cooperative and adaptable control solution, which can be tailored to the single user being able to follow his/her progress.

With this work, we first analyze the robot-mediated rehabilitation modalities proposed in the literature. We investigate the availability of low-level control strategies that can be exploited to promote the desired human-robot interaction behavior. Finally, we present the description of a low-level unified controller for upper-limb rehabilitation that is capable of assisting patients in a compliant manner and that promotes most of the robot-mediated training modalities used in clinics.

The fundamental concept of the here proposed approach relies on the availability of a unified compliant controller, which could change the level of assistance and resistance according to the patient's performances and contribution, towards the paradigms of personalization and continuity of care. Exploiting a unified controller might introduce some drawbacks, such as lower trajectory tracking accuracy. However, in rehabilitation robotics, precise positioning is not crucial because the robot is coupled with the human limb and should behave compliantly with it.

To test the proposed controller and its ability to render different human-robot interaction rehabilitation modalities, we conducted an observational study with healthy volunteers. The experiments consisted of one degree-of-freedom elbow flexion/extension movements, executed under seven different levels of assistance and resistance. We recorded the subject's voluntary effort through muscular activation signals (from biceps and triceps), and we measured the generated interaction torque at the elbow joint axis.

Structure of the work — This work is structured as follows. Section 2 defines the rehabilitation training modalities used in upper-limb robot-assisted therapy and their low-level control implementation challenges. The core idea of this work is presented in Section 3, which explains how high-level modalities have been integrated with a low-level unified compliant controller. Section 4 presents the experimental design implemented to test the controller, and the results are exposed in Section 5. Finally, Section 6 draws the discussion and conclusion of the work. 


\section{Related work and novel contribution}

Robot-mediated rehabilitation has been largely investigated since the 1990s. The literature agrees that the goal of robots should be to induce motor plasticity in subjects undergoing rehabilitation treatment and, therefore, to improve their motor recovery [29]. Therefore, robot-mediated control algorithms were designed and developed, taking inspiration from motor learning and neurophysiological aspects $[30,41,60]$. Consequently, different high-level training modalities were proposed to promote motor recovery at different stages of the disease. Such modalities are in turn embodied by low-level controllers that are capable of shaping the physical human-robot interaction according to the residual capabilities of the user,i.e., the aim of researchers is to design controllers able to minimize the interaction forces between the robot and the human while motivating the subject and guaranteeing the completion of the rehabilitation task. In other words, the robot should cooperate with the patient along with the rehabilitation treatment as a therapist would do, changing the levels of assistance, resistance, and motion correction based on the progression of the motor recovery. Over the past decade, several reviews on exoskeletal control for robot-assisted rehabilitation have been proposed in the literature. However, the researchers proposed several taxonomies and categorizations at various levels of abstraction $[5,24,45,51]$. In this work, we will use the term "training modalities" for "high-level" desired rehabilitation behaviors and the term "control strategies" for their "low-level" control scheme implementation. Generally, the training modalities for upper-limb rehabilitation are characterized by three main features: (i) corrective assistance, which implies that, given a pre-defined task, the system also corrects the movement when the subject moves away from the desired trajectory; (ii) weight counterbalance assistance, which refers to the ability of the robot to support and compensate the weight and the dynamics of the impaired limb; and (iii) resistance, which relates to training strategies that make the movement more difficult to perform, thus engaging the subject and stimulating the motor control learning process [58]. High-level therapy modes can also be divided according to the expected engagement and interaction between the subject and the robot. In literature, the taxonomy is not coherent and aligned, some works relate rehabilitation modalities to subject's status [5,58], others to the robot's desired behavior [45]. When describing the cooperation between robot and human, in this paper we propose a terminology that describes the expected subject's behavior during interaction. For example, "Passive mode" will refer to subject-passive/robot-active training.

On top of these general definitions, it can be observed that one of the most critical areas in rehabilitation robotics is implementing the desired high-level modalities within the robots hardware. In fact, regardless of the desired high-level behavior, the rehabilitation robot should first promote the so-called compliant motion, i.e., to behave transparently with respect to human activity.

Compliant motion, by definition, refers to the capability of the robotic system to generate movement and, simultaneously, undergo movement if external forces are applied. Namely, it permits power flow both from the actuator to the environment and vice-versa. In the first case, the actuator drives the motion and corrects for trajectory errors, while in the second case, the user is applying effort to the robot, and the robot has to permit deviations from a defined equilibrium point. The concept of compliant motion refers to the capability of a system to shape the dynamical relation between motion and torque/forces instead of independently controlling the joint motion or the joint torques of the robot. Typically, the perceived compliance can be implemented either through mechanical compliance, for example by using soft joints instead of rigid joints, or through compliant controllers $[8,35]$. Moreover, these approaches intrinsically improve back-drivability and safety during human-robot interaction [66]. 
From a low-level point of view, achieving compliant motion is a fundamental, yet challenging, task in rehabilitation robotics. In fact, if achieving rigid behavior of the robot can be considered a trivial task, obtaining its opposite can be challenging since the low-level controller should reject the dissipative effects introduced by the robot hardware. At the same time, one of the key characteristics of the motor recovery process is not to limit, in any way, any intention of movement coming from the user and, possibly, of guiding the subject's voluntary movements towards the correct task execution. Compliant motion in rehabilitation robotics can thus be addressed as a compromise between good trajectory tracking and minimization of interaction forces.

Usually, rehabilitation robots and exoskeletons are provided with high-ratio transmission gearboxes that are kinematically inefficient, and that can introduce static and viscous friction. In this scenario, the perceived compliance cannot be guaranteed by the back-drivability of the motor itself. Still, it can be implemented by adding an elastic element in series with the actuation unit, i.e., series elastic actuators (SEA) $[9,11,15,71]$, or with compliant controllers that add virtual springs and dampers to shape the virtual mechanical impedance at the joint.

In the literature, several low-level controllers have been proposed to achieve compliant motion, and in turn, to implement the previously described training modalities. Among all, impedance control is one of the most common approaches, and it has been demonstrated to be a very efficient solution for neurorehabilitation [48]. The impedance control belongs to those control schemes that permit a compliant physical human-robot interaction. It implements dynamic control that relates force/torque and position: a torque/force output is generated from a position input. In particular, impedance control is characterized by a nested loop architecture. An inner torque-feedback loop implements the transparent behavior and promotes the mechanical compliance (i.e., it "softens" the control). An outer position-feedback loop corrects for trajectory tracking errors by applying forces or torques aimed at the completion of the task (i.e., it "stiffens" the control). Furthermore, two different variants of the impedance control can be identified. When the actuation unit is inherently back-drivable, the torque control can be implemented through an open-loop current control loop (i.e., implicit impedance). In the other cases, a load-cell or an elastic element is exploited in series as a feedback signal for the closed-loop torque control loop (i.e., explicit impedance) $[8,63]$.

Regarding the rehabilitation domain, both impedance controllers in joint-space $[34,39,56]$ and in the Cartesian-space have been developed [20,43,44,62]. In joint-space impedance, the virtual mechanical elements are implemented in the joint-space with torsional spring and damper. The compliant behavior is given independently at each joint of the robot. Instead, with the Cartesianspace controller, virtual linear springs and dampers are connected to the robot end-effector in three-dimensional directions. Each direction is responsible for one of the three dimensions of the impedance ellipsoid computed at the robot end-effector. For example, in [39], the baseline low-level control strategy of the Harmony robot, which is a bimanual upper-body exoskeleton for poststroke rehabilitation, is based on a SEA-based joint-space impedance control that promotes the coordinated motion of the shoulder, through the assistance of the scapulohumeral rhythm [37]. Specifically, for each joint, the deformation of the elastic element is used to estimate the generated torque at the joint axis. Then, an outer position-feedback is added to correct for task deviation. The dynamic model of the exoskeleton is formulated with a recursive Euler-Newton algorithm, and a feedforward term is added to compensate for gravity, friction, and dynamic torques. Similarly, the ARMin exoskeleton $[23,34,54]$ is another example of an upper-limb exoskeleton for post-stroke rehabilitation based on a Proportional-Derivative (PD) position-feedback control that both supports the arm weight and provides assistance to the movement by virtually constraining the motion through stiffness/damping guidance. On top of this controller, the authors included online adaptive 
compensation algorithms to compensate for friction, elastic elements, and gravity terms [32,33]. On the other side, Frisoli and colleagues [20] developed a Cartesian-space impedance-controlled exoskeleton, able to discriminate the end-effector reference trajectory from its orthogonal trajectory. In detail, two concurrent low-level impedance controllers act along the tangential and orthogonal directions of the trajectory, providing different virtual stiffness levels along such directions and promoting a virtual tunnel that follows the Cartesian-space desired trajectory. Further evolution of impedance-based controllers involves the adaptation of the assistance according to the performances of the subject $[55,57]$. Proietti and colleagues [59] developed an exoskeleton controller based on adaptive techniques that can actively modulate the stiffness of the robotic device in function of the subject's activity. Instead, Pneu-WREX researchers developed a model-based adaptive control that learns from the patient's ability and provides support in completing movement while guaranteeing mechanical compliance [70]. They implemented a Cartesian-space impedance control law, to which they added a feedforward term characterized by a non-linear sliding mode control scheme. The assistance-as-needed adaptation was achieved by adding a learning factor, which iteratively corrects the feedforward contribution, and a force decay, which reduces the support when the subject is able to perform the movement correctly.

Nevertheless, when dealing with high-level rehabilitation modalities and their low-level control implementation, the literature is still swampy and fragmented. Firstly, most literature reviews focused on the desired rehabilitation behavior and did not investigate the implementation on the robots' hardware [45]. Secondly, the literature proposes several custom solutions that strongly depend on the kinematics, mechanics, and electronics of the developed robots. Finally, some research groups already proposed that a mixture of assistance, correction and resistance with impedancecontrol laws could be used to gradually increase the amount of expected voluntary muscle activity [16]. However, a generalization and validation of these approaches are still lacking.

With this work, we identified a cooperative control framework that implements multiple highlevel human-robot interaction modalities with a unified low-level explicit impedance control law. We will now describe and demonstrate its ability to promote different features, such as favoring good transparency of the joint, compensating for the weight of the robot and of the impaired limb, assisting the motion along the desired trajectory, recovering from task deviations, or challenging the user by applying resistance and increased gravity to the motion.

\section{Unified compliant control framework}

The unified controller relies on the concepts of compliant control and, in particular, impedance control. The overall scheme of the proposed controller is presented in Fig. 1. The virtual mechanical impedance is implemented in the outer position-loop $I(s)$, which is in charge of correcting for deviations from the desired angular position. Namely, it is driven by the difference between the commanded reference (i.e., $\theta_{r}$ ) and the measured angle (i.e., $\left.\theta_{l}\right)$. The inner torque-loop $F(s)$ is in charge of controlling the torque output at the load axis. Since its dynamics should not influence the outer loop, the inner loop is usually implemented at a higher control frequency. Thus, it is supposed to be fast enough so that its dynamics can be neglected with respect to the outer impedance loop.

In this work, we consider an exemplary single-degree-of-freedom joint, shown in Fig. 2, as a platform to validate the controller and its functionalities. The actuation chain is composed of an electric motor coupled with a high-ratio transmission gearbox. The unit is also provided with an incremental encoder that measures the joint angle, and a reaction torsional load-cell provides torque feedback at the output load axis. The dynamics of the one degree-of-freedom actuation system is 


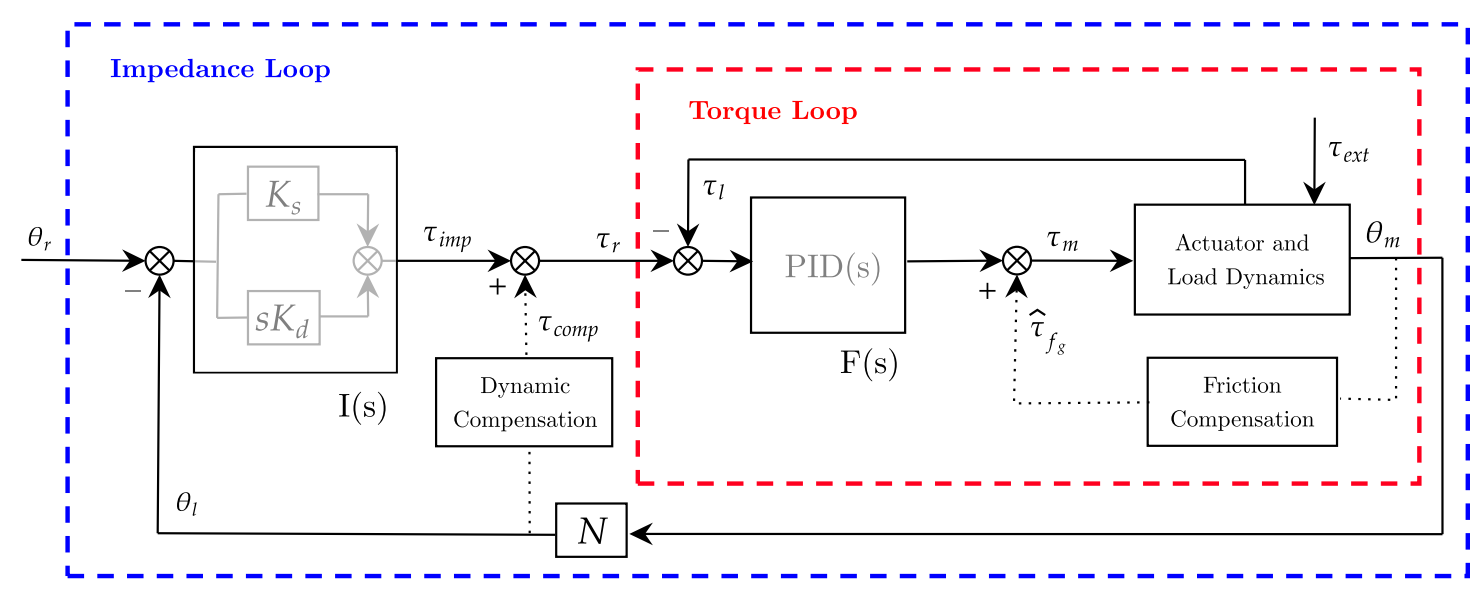

Figure 1: Block diagram of the unified controller scheme based on explicit impedance control law. Inner torque control $F(s)$ is in red and outer impedance control $I(s)$ is in blue. Dotted lines represent positive-feedback compensations.

as follows:

$$
\tau_{l}=\left(\tau_{m}-J_{m} \ddot{\theta}_{m}-\eta_{m} \dot{\theta}_{m}-\tau_{f g}\right) N
$$

where $\theta_{m}$ is the motor displacement, while $\tau_{m}$ and $\tau_{l}$ respectively represent the motor torque and the load torque measured at the load-cell. The generated motor torque $\tau_{m}$ is converted in the acceleration of the rotor $\left(\ddot{\theta}_{m}\right)$ with inertia $J_{m}$, in the dissipation of the motor damping $\eta_{m}$, and in friction $\tau_{f g}$ of the transmission gearbox. The resulting torque is then amplified by the gear ratio $N$ and transferred to the output axis $\left(\theta_{l}\right.$ of Figure 2$)$.

\subsection{Actuator torque control (Inner loop)}

The inner torque loop of an impedance controller can be implemented both as an open-loop (i.e., implicit impedance) or a closed-loop (i.e., explicit impedance) torque controller. In literature, Hogan first presented an implicit impedance controller that exploits an open-loop torque controller based on motor current control [27]. However, it requires inherent back-drivability, that can only be achieved with low-ratio transmission or direct-drive actuators [8]. Several other approaches are available to compensate for undesired gearbox inefficiency. Model-based force estimation [70] or disturbance observer-based control schemes [32] are common solutions. More often, torque sensors can be used to explicitly measure the actual generated torque and/or the subject's applied effort to be used as feedback in a closed-loop formulation $[18,47]$. In our work, since we consider high-ratio transmissions and the open-loop formulation would require a good friction model to achieve highfidelity torque control, we opted for torque-controlled joints that are provided with torsional loadcells at each joint. In fact, torque-controlled robots are capable of producing very low impedance, which is essential to encourage users' voluntary contribution. In this form, the inner torque control $F(s)$ is in charge of making sure that the measured torque output $\left(\tau_{l}\right)$ follows the outer loop control variable $\left(\tau_{r}\right)$. From the reference torque level to be actuated $\left(\tau_{r}\right)$, the inner torque-loop estimates the 


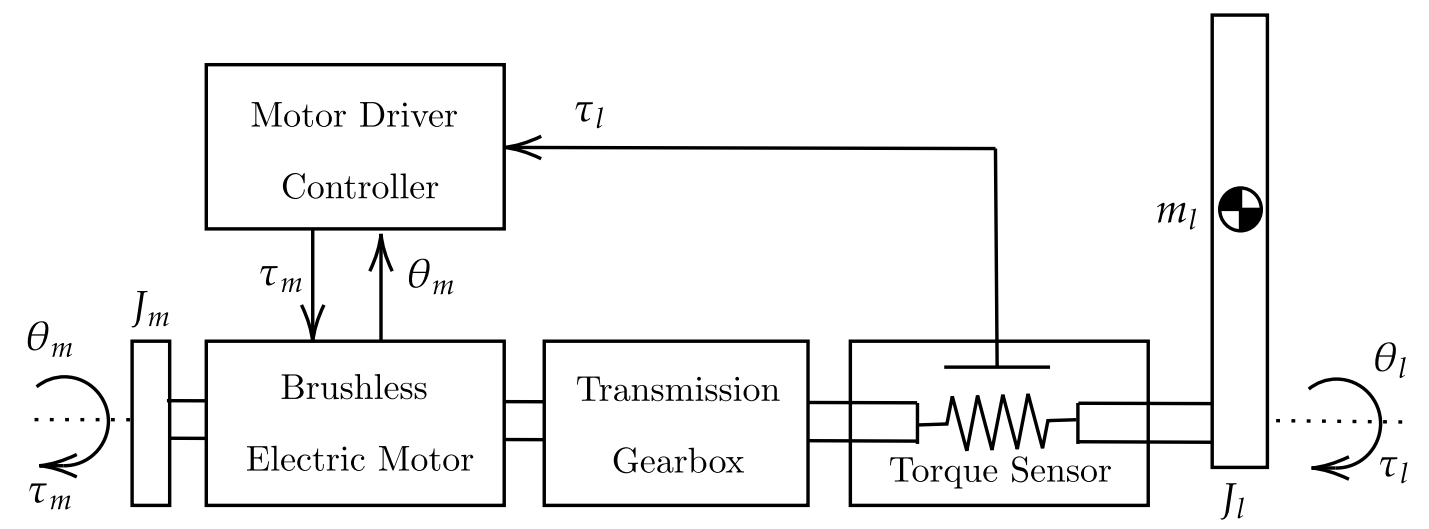

Figure 2: The actuation chain consists of an electric motor provided with an angular encoder, a transmission gearbox, a torsional torque sensor and an generalized aluminum bar load. The motor driver acquires input signals from the actuation chain and commands torque set-points to the electric motor.

target torque of the actuator $\left(\tau_{m}\right)$ through a Proportional-Integrative-Derivative (PID) controller, with feedback from the torsional load-cell $\left(\tau_{l}\right)$, that in the Laplace form is $(2)$ :

$$
F(s)=K_{p}+K_{i} / s+K_{d} s
$$

To compensate for static and viscous friction introduced by high-ratio gearboxes, an additional feedforward friction compensation $\left(\hat{\tau}_{f_{g}}\right)$, modeled as in [4], has been added at the inner loop level, as shown in Fig. 1. The compensation can be divided into Coulomb friction and velocity-dependent friction:

$$
\hat{\tau}_{f_{g}}=\tau_{c} * \tanh \left(\dot{\theta} / \dot{\theta}_{c}\right)+f_{v} \dot{\theta}
$$

where $\tau_{c}$ is the Coulomb friction torque, $\dot{\theta}$ is the measured joint velocity, $\dot{\theta}_{c}$ is the Coulomb joint velocity threshold, and $f_{v}$ is the viscous friction coefficient. The hyperbolic tangent function ensures the Coulomb term to be continuous and smooth across $\dot{\theta}=0$ in order to avoid undesired oscillations. The $\tau_{f_{g}}$ term is then combined with the PID torque output estimated $\tau_{m}$ term as input to the actuator. The actual torque actuated at the load axis is then measured by the load-cell $\left(\tau_{l}\right)$ and fed back to the PID controller to track the reference torque $\left(\tau_{r}\right)$.

\section{$3.2 \quad$ Impedance control (Outer loop)}

The impedance control can be regarded as an outer position loop that takes a reference in terms of angular position (i.e., $\theta_{r}$ ) and, by means of a virtual mechanical impedance, produces a reference torque (i.e., $\tau_{r}$ ) that in turn is fed to the inner control loop. The total reference torque can be seen as composed of two contributions, as in Eq. 4. The feedback impedance-based term, namely $\tau_{i m p}$, corrects for tracking errors and dampens undesired oscillations. The feedforward compensation term $\tau_{\text {comp }}$ accounts for the compensation for the dynamic model of the robot and for the weight counterbalance of the user's limb. 


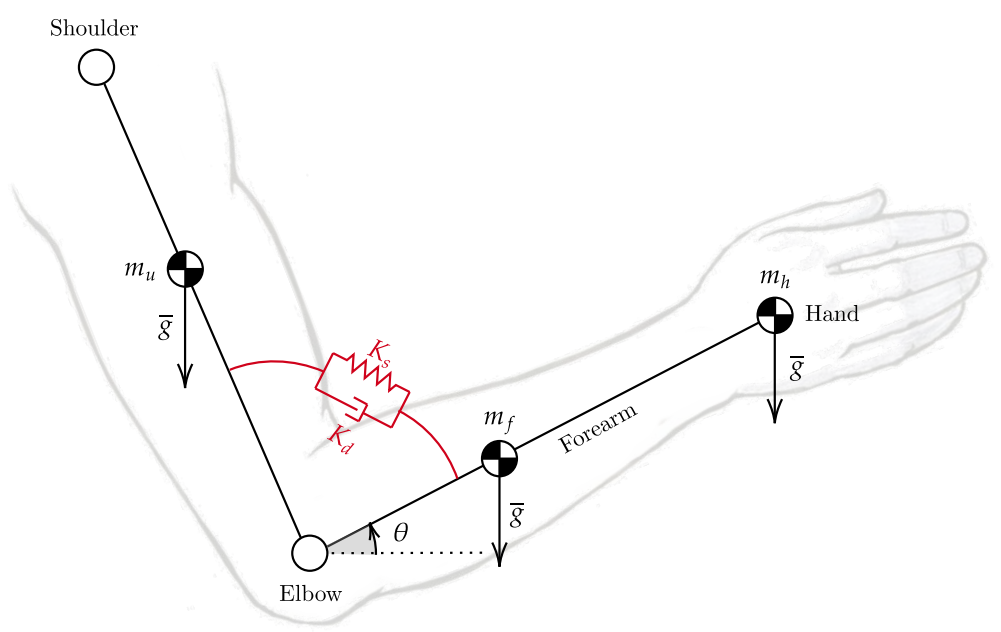

Figure 3: Impedance model at the elbow joint. The unified controller implements virtual stiffness and damping (in red) at the elbow joint. The elbow joint is rendered as a second-order system mass-spring-damper. The equilibrium point of the spring continuously changes according to the desired trajectory $(\theta)$.

$$
\tau_{r}=\tau_{i m p}+\tau_{\text {comp }}
$$

Instead, the measured torque at the load axis consists of the actual torque generated by the robotic system and can be broken down into four main components, as shown in (5):

$$
\tau_{l}=\tau_{\text {comp }}+\tau_{i m p}+\tau_{\text {ext }}+\tau_{\text {res }}
$$

where $\tau_{c o m p}$ and $\tau_{i m p}$ represent the actuation torques commanded to the motor as in Eq. $4, \tau_{\text {ext }}$ is composed of the external torque that the user exerts to the motor, and $\tau_{r} e s$ represents the residual disturbance torque that the inner torque controller can not reject.

\subsubsection{Feedback impedance-based term}

To derive the feedback impedance-based term (i.e., $\tau_{i m p}$ ), considering a first order impedance, the transfer function I(s) between the reference target $\left(\theta_{s}\right)$ and the impedance-based torque term $\left(\tau_{\text {imp }}\right)$ is characterized by two parameters: virtual spring $\left(K_{s}\right)$ and virtual damper $\left(K_{d}\right)$, and it can be implemented in the well-know form (6):

$$
I(s)=K_{s}+s K_{d}
$$

that in the time domain becomes (7):

$$
\tau_{i m p}=K_{s}\left(\theta_{d}-\theta\right)+K_{d}\left(\dot{\theta}_{d}-\dot{\theta}\right)
$$

where $\tau_{i m p}$ is the desired impedance control torque that is used as a set-point by the inner torque loop, while $\theta_{d}$ and $\theta$ are, respectively, the desired and measured joint angle positions. 
The virtual stiffness, by means of the virtual spring constant $K_{s}$, pulls the joint link towards its desired configuration (i.e., the spring corrects for deviations from its equilibrium point, which is continuously adapted to follow the desired angular trajectory). At the same time, the virtual damper $\left(K_{d}\right)$ dissipates the spring energy and damps oscillations. Overall, the role of these parameters is to render, as shown in Figure 3 for the elbow joint, a second-order system by virtualizing a springdamper component within the impedance control law. When dealing with the robotic rehabilitation, the desired angular velocity might not be available, especially when the task trajectory is updated in real-time to follow the subject's intention of movement. In such cases, we can neglect the reference velocity term $\left(\dot{\theta}_{d}\right)$ in the previous equation (7). In this way, the damping term is related to the absolute velocity instead of the error velocity. The virtual damper is fixed to the ground frame, resulting in always-resistive damping of the system.

\subsubsection{Feedforward weight and dynamics compensation term}

A feedforward torque reference term that accounts for the dynamics of the robot and for the weight of the arm is added at the impedance control level.

For the sake of simplicity, in this section, we consider the general single-degree-of-freedom joint shown in Fig. 3, which can be reduced to a rigid pendulum system. The torque acting at the load axis can be described with the dynamic equation of the system, which includes both the robot and the human, as follows (8):

$$
\tau=J_{l} \ddot{\theta}+f_{l} \dot{\theta}+G(\theta)
$$

where $J_{l}$ is the inertia moment, $f_{l}$ is the viscous friction at the load axis, and $G$ represents gravitational torques for both the link and the forearm. Compensating for the inertial component of the dynamic model requires the estimation of inertia moments and the computation of the acceleration by twice-differentiating the encoder position. These operations can raise many difficulties and undesired uncertainties that are in turn fed to the controller as positive-feedback terms. Inertia compensation can thus make the system non-passive and can jeopardize the coupled stability of the human-exoskeleton system [38]. Additionally, in robotic rehabilitation, the desired arm movements are usually slow, leading to neglectable effects due to the dynamic terms of Eq. 8. For these reasons, in our work, we only compensate for gravitational and viscous frictional torques.

We therefore introduce the simplified compensation term:

$$
\tau_{c o m p}=\hat{f}_{l} \dot{\theta}+\hat{G}_{l i n k}(\theta)+\hat{G}_{w c}(\theta)
$$

where $\hat{f}_{l}$ is the estimated viscous friction coefficient, $\hat{G}_{l i n k}$ represents the weight compensation term for the robot components, and $\hat{G_{w}} c$ represents the weight compensation of the human component. The weight compensation term for the robot can be modeled as in (10):

$$
\hat{G}_{l i n k}(\theta)=m g l \cos \theta
$$

where $m$ is the robot link mass, $l$ its center-of-mass distance, and $g$ is the gravitational acceleration.

As for the gravitational compensation term of the human $\left(\hat{G}_{w c}\right)$, we need to make explicit reference to the single-degree-of-freedom joint used as a demonstrative example (Fig. 3). Of course, this can be generalized to any joint of interest. We have included vertical forces applied at the centers of mass of the forearm and hand. The position of the center of mass and the weight of the limbs can be derived from the anthropometric tables presented in [69]. The level of weight assistance can be 
regulated by means of a weighting factor (ranging from $0 \%$ to $100 \%$ ) that accounts for misalignment and uncertainties in the anthropometric data as in (11):

$$
\hat{G}_{w c}(\theta)=W\left(\hat{m}_{f} l_{f}+\hat{m}_{h} l_{h}\right) g \cos \theta
$$

where $W$ is the weighting factor, $m_{f}$ and $m_{h}$ are the masses of forearm and hand, while $l_{f}$ and $l_{h}$ are their centers of mass. With this dynamic compensation, only inertial, centrifugal, and residual frictional torques are to be overcome if the user wants to perform a voluntary movement (i.e., they are not included in the compensation term).

The feedforward compensation torque formulation can be obviously generalized if a $n$-degreeof-freedom robot is concerned.

In this case, the dynamics compensation terms can also include Coriolis and Centrifugal torques. Such feedforward compensation can be computed from centralized inverse dynamics algorithms, such as closed-form solutions or more computationally efficient recursive Euler-Newton approaches $[14,64]$.

\subsection{Human-robot interaction rehabilitation modalities}

The literature proposed several approaches and control modalities for robot-mediated therapy towards the goal of personalized therapy. In this work, we included seven high-level human-robot interaction modalities, ranging from those that assist the movement the most to challenging strategies. In this section, we first describe the desired behavior for each of the proposed modalities. Then, we propose a match between the high-level behavior and a set of parameters for the presented low-level unified controller that can render the desired behavior.

Passive mode (P) The Passive mode should be exploited during the preliminary stages of the rehabilitation process. The robot helps the patient to track a predefined trajectory to improve the limb range of motion and reduce muscular atrophy or tendon retractions [46]. When the system is operated in Passive mode, the robot performs the movement without accounting for the user's intentional activity. Impedance control gains are greater than in other modes, rendering a stiffer behavior of the joint, and the torque feedforward term $\left(\tau_{\text {comp }}\right)$ is used to compensate for the user's arm weight. However, in this mode, the trajectory tracking is not as accurate as in position control, as the impedance control intrinsically introduces a tolerance dead-band. Nevertheless, this is not a critical aspect for rehabilitation robots since the crucial feature is to limit the interaction forces with the human limb.

Active Assistive modes Active assistive modes should be used when patients have some voluntary muscular contractions, but the generated strength is not sufficient to perform complete and functional movements. The robot provides the assistance needed to fulfill the task [5]. In these modes, subjects generate the minimum effort needed to accomplish the desired task. The user is actively involved in the movement, and the robot partially assists the motion. As mentioned before, we can further divide the robot-assistance into two different contributions: impedance-based corrective assistance, which provides a torque gradient to correct for angular trajectory errors, and weight counterbalance assistance, which compensates for the limb weight [45]. By combining these contributions, we can implement three different training modalities: 
Active Corrective mode with Weight Counterbalance $(\mathbf{C}+\mathbf{W})$ The $C+W$ mode is provided with both the impedance-based assistance with $\tau_{i m p}$ and the counterbalancing assistance with $\tau_{c o m p}$. Indeed, the control law always supports the arm weight and assists the user when the voluntary movement is not accurate, i.e., when the arm deviates from the pre-defined trajectory.

Active Corrective mode without Weight Counterbalance (C) The $C$ corrective mode is implemented with the impedance-based assistance. One can expect that this mode should render a similar behavior to the previous modality. However, when the feedforward weight compensation is not included, more effort is expected from postural muscles during tasks against gravity, while less antagonistic contraction could be needed in movements in favor of gravity.

Active Weight counterbalance mode (W) The Active Weight counterbalance mode can be applied to perceive a microgravity environment. This effect is obtained through the counterbalancing assistance term that is computed according to the configuration of the user's arm. In this modality, the subject is wholly involved in the task, and if the voluntary activity is not sufficient to fulfill the exercise, the robot does not apply for any corrective assistance. Indeed, the controller is not aware of the predefined exercise task a priori. At low-level, the virtual stiffness is removed, and low damping is kept to avoid undesired oscillations and dampen the motion.

Active Transparent mode (T) In Active Transparent mode, the user performs the task, and the robot follows the movement without assisting (nor resisting) the movement. In other words, this modality enables the robot to be dynamically transparent to users' voluntary movements, by compensating the exoskeleton weight at each configuration along the task.

Regarding its implementation, the low impedance behavior is achieved by means of a zerotorque controller provided only with the compensation for the robot dynamics. Neither assistance nor resistance is provided.

Resistive mode (R) The Resistive mode has been introduced to further engage the patient along his/her progression, i.e., when most of the motor functionalities have been (hopefully) relearned, but the subject still has to gain some muscular tone. In fact, robots with torque-controlled joints can also realize an aquatic therapy-like environment by providing weight support and allowing user-driven free motions with or without viscous resistance [40,65]. To implement such a behavior, this modality adds a viscous-like resistance to the movement while compensating for the robot dynamics. No impedance-based assistance is present, and the controller resists the movement by applying a viscous frictional torque, which is inversely proportional to the movement velocity.

Challenging mode $(\mathbf{C h}) \quad$ The Challenging mode amplifies the effect of gravity during the movement. This modality can be used to challenge the subject during the exercise and to focus the training on postural anti-gravity muscles. In particular, instead of counterbalancing the limb weight, the controller adds additional virtual weight, applied at the centers of mass of the limb, that gives the feeling of doing the task with weight, or, in other words, of doing the exercise in a hyper-gravity environment.

Overall, qualitative guidelines suggest that high-impedance implementation should be used to stiffen the control law, thus imposing the subject's movement along the task trajectory, while low- 
impedance gains should be exploited to render more compliant and softer behavior of the robot, i.e., the controller promotes voluntary movements, and the user is allowed to deviate from the trajectory. Finally, large damping values can reduce oscillations and dissipate energy. However, a trade-off in the impedance parameters is needed to induce a physiological muscular activation aimed at completing the task in an assisted-as-needed fashion.

To define the quantitative values of stiffness, damping and weight assistance for each modality, we separately ran some preliminary tests on two healthy subjects, which were not recruited for the rehabilitation modalities assessment to avoid bias. The parameters of the controller were empirically determined according to the perceived behavior. The damping $K_{d}$ is kept proportional to the stiffness $K_{s}$ to avoid undesired oscillations and jerky movements as:

$$
K_{s}=\alpha K_{d}
$$

where the ratio factor $\alpha=5$.

\subsection{Stability and passivity considerations}

To guarantee safety in human-robot interaction, the coupled stability of the human-robot system is a fundamental requirement [10]. In particular, given two separately stable systems, the coupled stability is always guaranteed. However, an unstable robotic system can become stable after coupling it with the human limb [13]. Such conditions can be derived from analyzing the passivity of the interaction port of the robot (i.e., where effort and velocity flow from the robot to the subject and vice-versa) [25]. In our case, besides the loose and flexible attachment between the forearm and the cuff, we consider a rigid human-robot interaction port and a nearly passive behavior of the human arm [28]. The coupled stability of the human-robot system is thus guaranteed by assessing the passivity of the controlled robotic system. Considering stiff joints, several stability analyses have been conducted for implicit impedance control schemes, and the range of impedances, namely Z-width, that can be rendered by passive compliant controllers, have been presented for different control methods [12]. Usually, authors provide boundary conditions, in terms of controller parameters, that make the robotic system to behave passively. Contrarily, explicit impedance control schemes are more recent and no analytical formulation is available yet [8]. Recently, Focchi and colleagues experimentally showed that the range of impedance parameters (Z-width) is strongly affected by the bandwidth of the inner nested loops [18]. They also demonstrated that achieving higher rendered impedance could be possible by using faster actuator bandwidth, or by lowering gains of the inner torque loop $[6,7]$.

Furthermore, in our work, given the dynamics of the reaction torque sensor, the gearbox nonlinear dynamics and the non-modeled backlash of the gearbox, we should model the robot with a flexible joint. According to Calanca et al. [8], flexible joints are soft joints in which the elastic and/or damping elements are not included on purpose, but they arise from undesired effects due to the transmission chain. This leads to a slightly different impedance control scheme, where the position feedback is collocated with the motor actuation, while the torque feedback is non-collocated, conceptually similar to the control of a SEA model, but with higher stiffness and damping physical parameters [1]. Also in this case, it is suggested that "there is a trade-off between achievable stiffness and low undesired interaction torques" [66] and that "a desired stiffness higher than the physical spring stiffness is not allowed for passivity" [9]. Given the above mentioned considerations, it is clear that, even for simple controllers, the passivity-based control design problem is non-trivial. 
High control bandwidth for the torque control loop (i.e., having a high-fidelity torque tracking) might not be beneficial for achieving stability and passivity. For these reasons, in this work we did not explicitly investigated the coupled stability, nor we analytically derived its passivity, but we followed qualitative guidelines to empirically tune the control parameters and display a stable behavior of the coupled system.

\section{Methods}

To assess the validity of the proposed control framework and its ability to promote different humanrobot interaction modalities, we considered a typical actuation joint for a general upper-limb exoskeleton, and we used it as a platform to test and verify the performances of the proposed controller.

\subsection{Experimental set-up}

The actuation is provided by a brushless DC motor (EC-45 flat, Maxon Motor AG, Switzerland), coupled with a planetary gearhead with a transmission ratio of 156:1 (GP-42-C, Maxon Motor AG, Switzerland). The electric motor provides a nominal torque of about $120 \mathrm{mNm}$. Thus, given the ratio and the efficiency of the transmission, the gear motor is able to provide at the load side a maximum constant torque of about $15 \mathrm{Nm}$ and a peak torque of about $18.5 \mathrm{Nm}$. An incremental encoder reads the rotor position with a resolution of 2048 counts per revolution, leading to a resolution of $0.001^{\circ}$ at the load side. Finally, a reaction torsional load-cell (TRT-200, Transducer Techniques, CA, USA) is connected to the gearbox output shaft to sense the torque acting on the joint of the robot. With the aim of testing the control in the interaction with the human motion, we designed a one-degree-of-freedom robotic system to provide assistance to the elbow during flexionextension tasks. In particular, the rotational axis of the system is aligned with the user's elbow joint. An aluminum bar is attached to the load-cell and is fixed to the user's forearm by means of an ergonomic arm cuff. The arm cuff is equipped with padded fabric which minimizes interaction forces between the rigid shell and the arm. Adhesive strips are used to fix it to the arm cuff. The user's elbow rests over a soft foam surface, and the arm cuff position can be adjusted according to the forearm length to improve the comfort and alignment of the rotation axis. The unified controller described in Section 3 is implemented in a real-time control system, based on Linux patched with PREEMPT RT, and runs at a cycle time of $1 \mathrm{~ms}$. The control hardware architecture shown in Fig. 5 relies on the EtherCAT field-bus, which guarantees good performances on distributed networks, and assures a reliable, deterministic, stable, and low-latency communication between the control unit and the connected hardware. In particular, the motor driver (Mini Torque Driver, Esmacat, US) is connected to the control system via the EtherCAT communication, and a real-time $\mathrm{C}++$ master application, based on the Simple Open-Source EtherCAT Master (SOEM) library, is implemented to handle the communication with the motor and sensors. The real-time control unit also implements the outer impedance position loop at $1 \mathrm{kHz}$, the feedforward compensations, and the trajectory generation. The low-level torque control is instead implemented in the motor driver at $5 \mathrm{kHz}$.

The experimental set-up and its connection are described in Fig. 4b, while its final realization is shown in Fig. 4a. The main features of the presented experimental set-up are reported in Table 1 . 


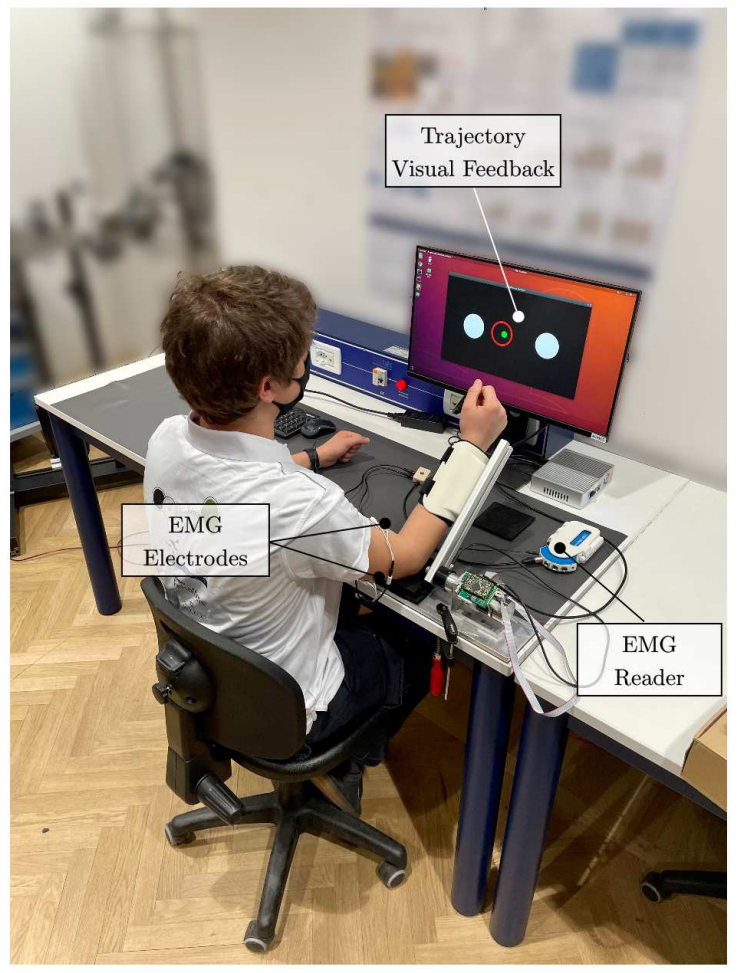

(a) Arm configuration

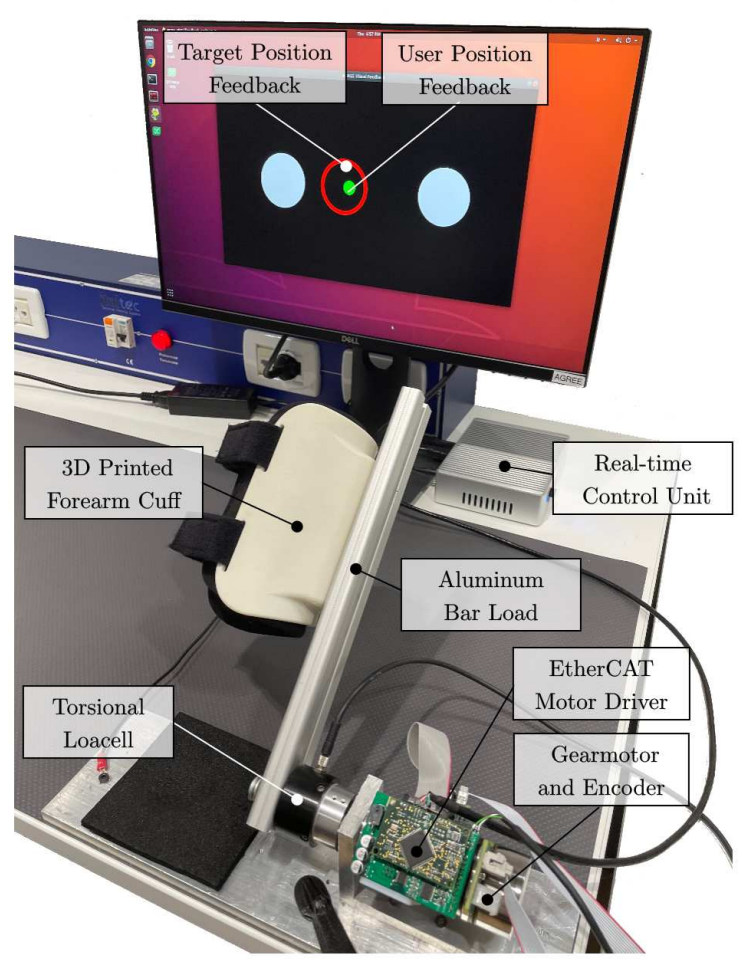

(b) Experimental setup

Figure 4: (a) The subject is attached to the elbow-joint system at the forearm. The elbow leans on the table over a soft foam surface. Surface electrodes are placed at the biceps and triceps brachii (long head). (b) The actuation drive system is connected to the real-time control unit with EtherCAT. Visual feedback is provided to the user to help follow the desired trajectory.

\subsection{Actuation and control assessment}

The performances of the proposed unified control framework have been evaluated using the following metrics. Firstly, we assessed the capability of the system to promote physical human-robot transparency, defined in literature as how good the robot is at rejecting torque disturbances and at limiting resistance during subjects' voluntary motion [72]. To this aim, we asked to a healthy subject to perform movements with elbow one-degree-of-freedom robot in Transparent mode in two conditions: i) spanning the whole available range of motion (i.e., $-90^{\circ}$ to $+90^{\circ}$ ) in free-motion at various velocities ranging from -0.1 to $0.1 \mathrm{rad} / \mathrm{s}$; ii) tracking a pre-defined minimum-jerk trajectory shown on the screen at various velocities ranging from -0.1 to $0.1 \mathrm{rad} / \mathrm{s}$. We measured the torque output from the load-cell sensor, while the position and velocity of the joint axis were obtained from the incremental encoder. To evaluate the back-drivability of the controlled joint, we computed maximum residual resistive torques, which should be minimal for better rendered transparency. To assess the accuracy of the torque control, we analyzed the frequency response of the inner closed-loop. We set the drive system at its mechanical end-stop, and we commanded 


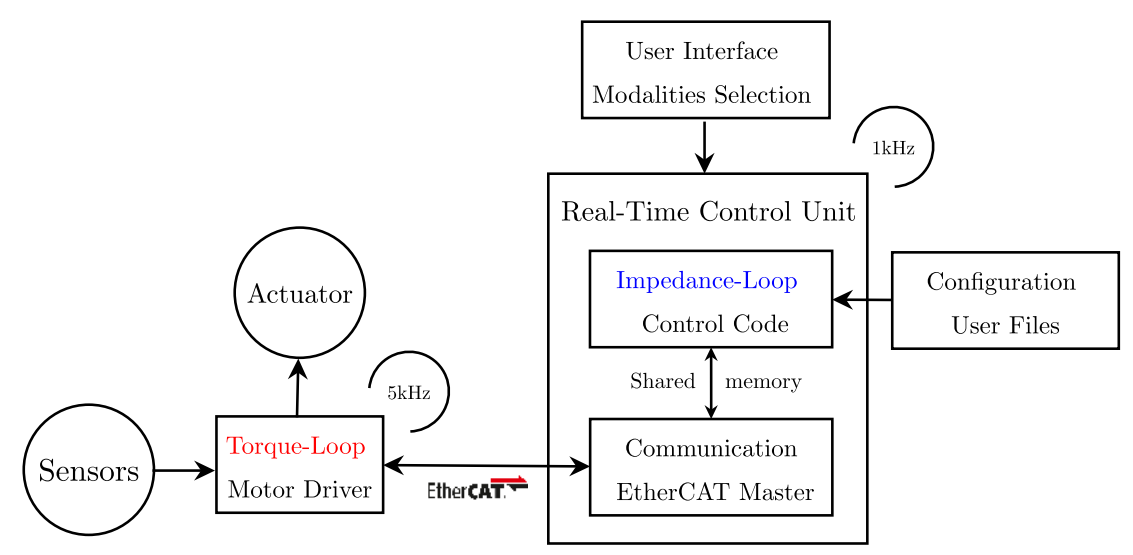

Figure 5: Control framework block diagram. The inner torque-loop is implemented in the motor driver. The outer impedance-loop is implemented in the real-time control unit. Configuration files are used to personalize the controller parameters. The user can select the rehabilitation modality through a simple user interface.

\begin{tabular}{c|c}
\hline \hline Specifications & Value \\
\hline Nominal Torque & $15 \mathrm{Nm}$ \\
Max. Peak Torque & $18.5 \mathrm{Nm}$ \\
Max. Velocity & $4.4 \mathrm{rad} / \mathrm{s}$ \\
Gearbox ratio $(N)$ & $156: 1$ \\
Range-of-Motion & $-90^{\circ}+90^{\circ}$ \\
Torque Control Frequency & $5 \mathrm{kHz}$ \\
Impedance Control Frequency & $1 \mathrm{kHz}$ \\
\hline \hline
\end{tabular}

Table 1: The main features and specifications of the developed experimental set-up. Performances of the torque control and impedance control loops are discussed in Section 5.1. 
sinusoidal torque profiles to the actuator torque control at different frequencies, ranging from 0.5 $\mathrm{Hz}$ to $4 \mathrm{~Hz}$. We evaluated the differences between the commanded torque and the measured torque curves using Root Mean Squared Error (RMSE) and Peak Error (PE) values, which are measures of the accuracy of the torque-control loop and both should be as small as possible. Finally, we computed the Pearson correlation coefficients to evaluate torque fidelity at each frequency, which should be greater than $90 \%$ for high similarity levels [52]. Finally, we investigated the performance of the impedance control, and we estimated the accuracy of the rendered torsional impedance values that the system was able to generate. The robot was commanded in impedance at the vertical equilibrium point $\left(\theta=0^{\circ}\right)$, and external torques were exerted to the joint-link system. The experiment was repeated at different stiffness/damping values. The displacement from the equilibrium point (in radians) at stiffness values of $5,10,20$, and $40 \mathrm{Nm} / \mathrm{rad}$ has been evaluated and related to the measured torque output. One should verify that the experimental stiffness matches the commanded one.

\subsection{Human-robot interaction modalities assessment}

The testing protocol was performed on healthy subjects, and it as approved by the ethical committee of Politecnico di Milano. The protocol involved the execution of elbow flexion/extension tasks with the elbow-joint developed set-up (Section 4.1). The system was connected to the dominant arm of the user, and the user performed elbow flexion and extension movements following the seven implemented rehabilitation strategies. Their sequence was randomized to avoid learning or fatigue effects, that could have biased the results. For each modality, the user performed 15 elbow-extension repetitions. The user was instructed to perform the movements following a visual feedback (Fig. 4a). The visual interface showed the movement to be tracked and the actual position of the joint. The desired movement speed was kept the same across all modalities.

As proposed in [26], the movement of the human arm, when coupled with a robot, can be described by a minimum jerk trajectory. In this work, we defined the nominal trajectory by means of a symmetric fifth order $\beta$-function [42] as in Equation 13. The nominal trajectory starts with the forearm lying on the table (i.e., $0^{\circ}$ ), then the flexion/extension movement is performed in about 8 seconds as in Fig. 6a.

$$
\begin{gathered}
\theta_{r}(t)=P_{0}+P_{1}\left(t-P_{2}\right)^{P_{3}}\left(P_{4}-t\right)^{P_{5}}, P_{2} \leq t \leq P_{4} \\
P_{1}=\frac{A_{0}}{\frac{P_{4}-P_{2}}{2}\left(P_{3}+P_{5}\right)}
\end{gathered}
$$

where the $P_{n}$ parameters are used to configure the desired trajectory. $P_{0}$ represents the initial position offset, $P_{2}$ and $P_{4}$ are the start and the stop time, $P_{3}$ and $P_{5}$ are the interpolators' orders for the raising and falling phases, and $P_{1}$ is related to movement amplitude $A_{0}$ by means of Eq. 14 . Fig. $6 \mathrm{~b}$ shows the desired $\beta$-function trajectory for the elbow flexion/extension movement.

\subsubsection{Outcome measures}

We recorded the kinematic and dynamic data from the robot sensors. Commanded and measured angular position, velocity and torques were sampled at a frequency of $1 \mathrm{kHz}$. Torque data were low-pass filtered with a Butterworth filter of the third order and a cut-off frequency of $20 \mathrm{~Hz}$. To investigate how subjects adapted their motion control to various assistance (or resistance) levels, 


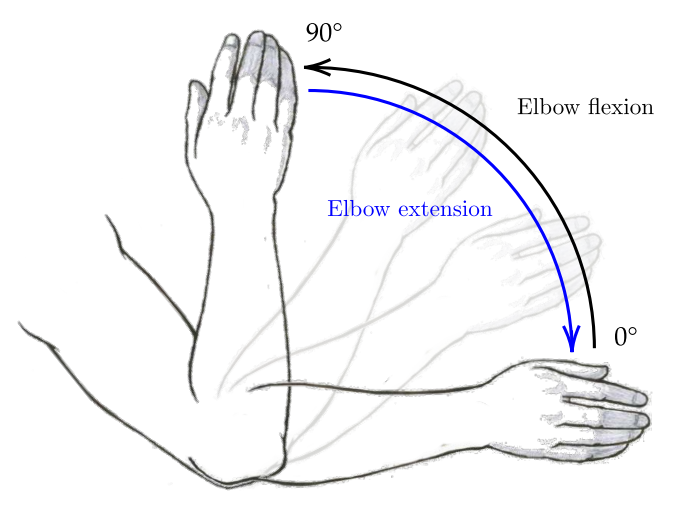

(a) Elbow flexion/extension movement
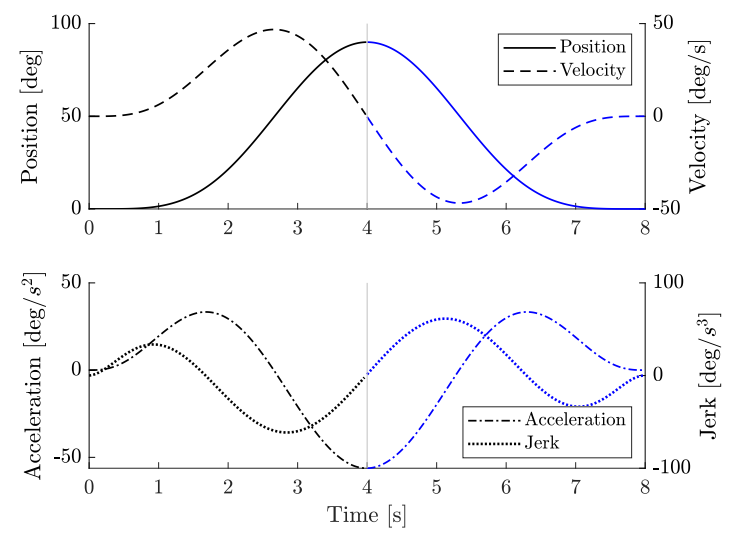

(b) $\beta$-function task trajectory

Figure 6: (a) Sketch representation of the elbow flexion/extension task exercise. (b) Trajectory $\beta$-function computed with $P 0=P 2=0, P 4=8, A 0=90^{\circ}$, and $P 3=P 5=5$. Black lines represent elbow flexion phase, while blue lines represent elbow extension phase.

and to posit if the experiments were comparable, we assessed the kinematics variability. In particular, to evaluate if the subjects performed comparable trajectories across all modalities and, as a consequence, if we could posit that all the subjects performed the same movements, we computed the Root Mean Square Error (RMSE) between the commanded and the measured angular position across all repetitions, subjects, and modalities.

To validate the implemented control strategies and to investigate how they affect the user's behavior, we also registered the muscular activity. In particular, we recorded the biceps and triceps (long head) muscles, as shown in Fig. 4b . The superficial electromyographic signal (sEMG) was recorded at a frequency of $1 \mathrm{kHz}$ with a wireless EMG reader (Sessantaquattro, OTbioelettronica, Italy). EMG signals were pre-processed following a standard approach that includes high-pass filtering with a third order Butterworth filter at $10 \mathrm{~Hz}$, rectification, and low-pass filtering with a third order Butterworth filter at $4 \mathrm{~Hz}[21]$. We normalized signals for each participant with respect to the $80 \%$ of the maximum contraction during the whole experimental session, preventing normalization by spurious EMG spikes [61]. We computed the integrated EMG (iEMG) as marker of voluntary muscle drive as the area under the curve of the normalized EMG signal [3].

\subsubsection{Statistical analysis}

Outcome measures were collected for each subject and for each control modality. All output indices were computed separately for the flexion and extension movements. The results are expressed as median [25th - 75th quartile]. Given the reduced sample size, the Friedman test was performed to detect possible significant changes in the RMSE and iEMG indices across different control strategies. Post-hoc comparisons with Bonferroni correction were used to identify statistically significant differences between the seven modalities. All statistical analyses have been performed in MATLAB (version R2020b) and IBM SPSS Statistics (version 27). 


\section{$5 \quad$ Results}

\subsection{Actuation and control results}

As for the capability of the system to promote physical human-robot transparency, results demonstrated that the torque controller accurately followed the commanded torque (i.e., the anti-gravity torque) in both dynamic conditions. The maximum residual resistive torque during back-driving movements was about $\pm 0.3 \mathrm{Nm}$, which was perceived as negligible by the user that was performing the experiment. As shown in Figs. 7a and 7b, the robot confirmed good transparency and low impedance behavior within a range of $-1.0 \mathrm{rad} / \mathrm{s}$ to $1.0 \mathrm{rad} / \mathrm{s}$, which are typical maximum velocities for a rehabilitation exercise.
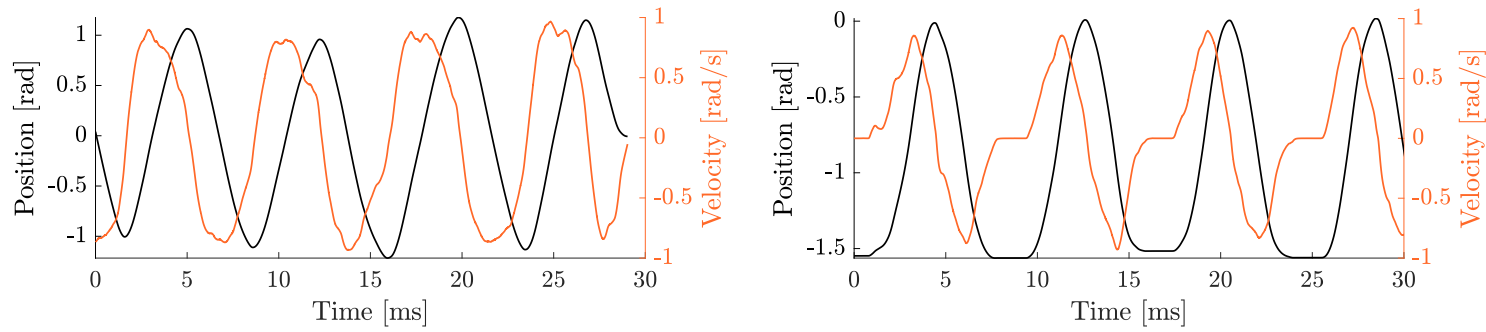

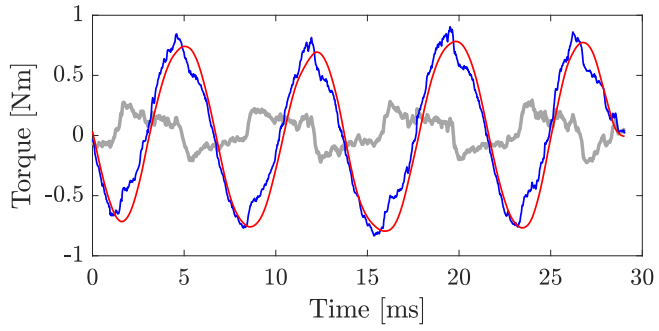

(a) Free-motion backdrivability

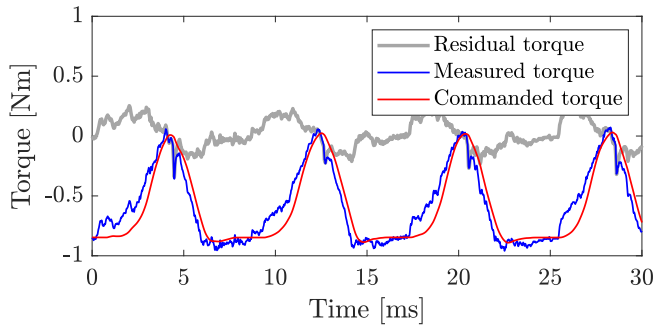

(b) Trajectory backdrivability

Figure 7: Backdriving movements in Transparent mode during free-motion (a) and following a predefined trajectory (b). Transparency was tested at velocities ranging from -1.0 to $1.0 \mathrm{rad} / \mathrm{s}$. The commanded torque compensates for the robot weight (i.e., gravity torque). The residual torque represents the difference between commanded and measured torque. For both experiments the residual torque ranges from about -0.3 to $0.3 \mathrm{Nm}$

The differences between the commanded torque and the measured torque curves were computed to assess the accuracy of the inner closed-loop torque control. Results showed torque output RMSE of $0.12,0.30,0.33$ and $0.49 \mathrm{Nm}$ respectively for $0.5,1.0,2.0$ and $4.0 \mathrm{~Hz}$. The maximum Peak Error (PE) of about $0.90 \mathrm{Nm}$ was obtained at $4.0 \mathrm{~Hz}$ in correspondence of sudden changes (i.e., at the inversion of velocity). Pearson correlation coefficients resulted equal to $99.62 \%$ ( $\mathrm{f}=0.5 \mathrm{~Hz}$ ), $98.06 \%$ $(\mathrm{f}=1 \mathrm{~Hz}), 97.55 \%(\mathrm{f}=2 \mathrm{~Hz})$ and $94.71 \%(\mathrm{f}=4 \mathrm{~Hz})$, demonstrating a high-fidelity torque control.

As for the performances of the impedance controller, Fig. 9 shows the relationship between the generated torque output (in Nm) and the displacement from the equilibrium point (in rad) at stiffness values of $5,10,20$, and $40 \mathrm{Nm} / \mathrm{rad}$. Notably, the fitted values from the experimental data 

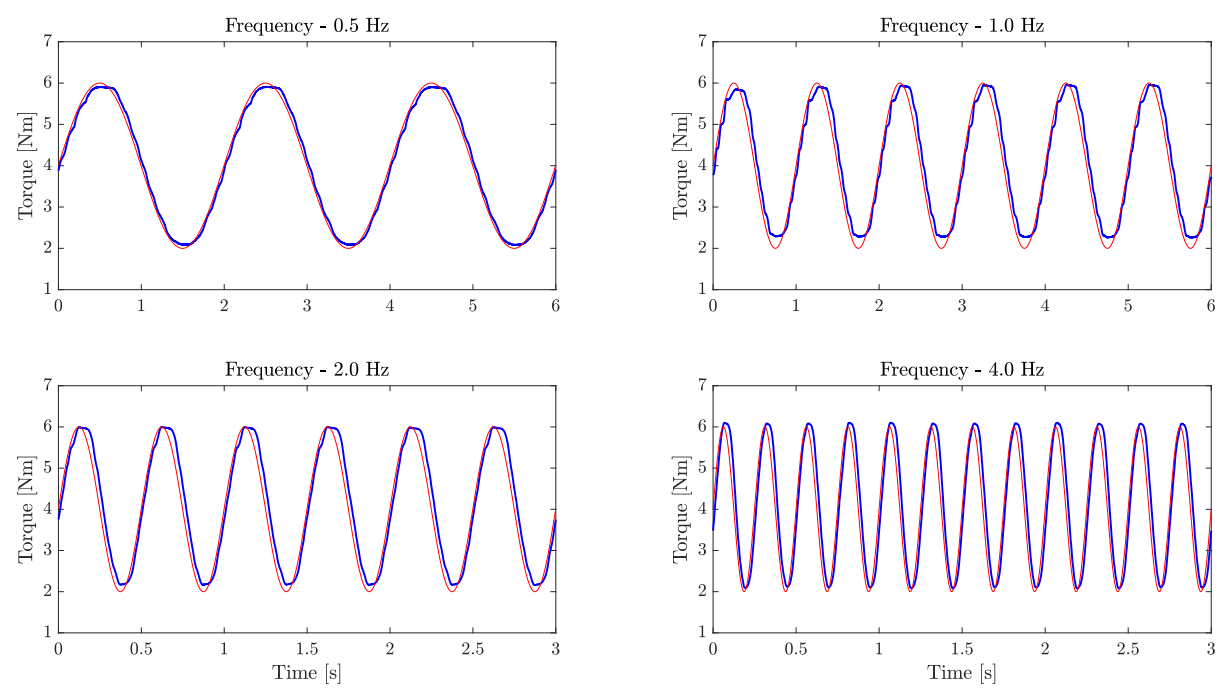

Figure 8: Sinusoidal torque response at different frequencies: 0.5, 1.0, 2.0 and $4.0 \mathrm{~Hz}$. The light red line represents the commanded torque, while the bold blue line refers to the load-cell measured torque.

demonstrate a good stiffness accuracy, resulting in an average relative error of $3.3 \% \pm 0.3 \%$ with respect to desired values.

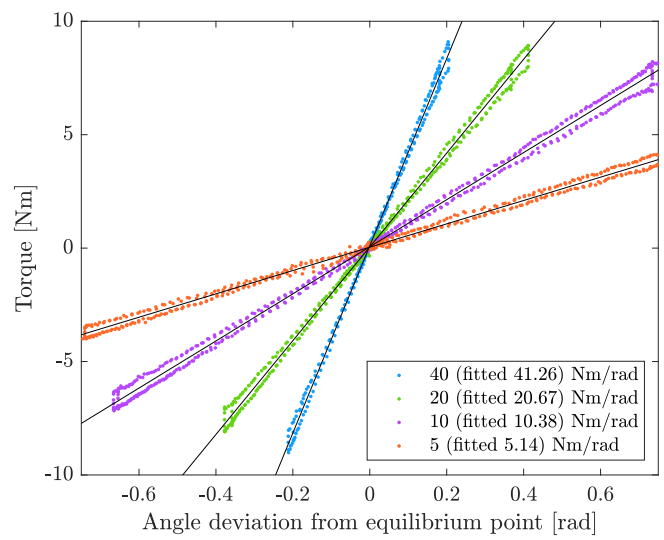

Figure 9: Experimental torsional stiffness. The joint is controlled in impedance at the equilibrium point $\left(\theta=0^{\circ}\right)$. The user exerts external forces to the joint. Torque and angle deviation are measured and compared at different virtual stiffnesses. For the commanded values of 5, 10, 20 and $40 \mathrm{Nm} / \mathrm{rad}$ the measured experimental stiffnesses are respectively 5.14, 10.38, 20.67 and $41.26 \mathrm{Nm} / \mathrm{rad}$. 


\begin{tabular}{c|c||c|c|c}
\hline \hline Rehabilitation Modalities & Mode & Weight $(W)$ & $\begin{array}{c}\text { Stiffness }\left(K_{s}\right) \\
{[\mathrm{Nm} / \mathrm{rad}]}\end{array}$ & $\begin{array}{c}\text { Damping }\left(K_{d}\right) \\
{\left[\mathrm{Nm} / \mathrm{rad}^{2}\right]}\end{array}$ \\
\hline Passive & $\mathrm{P}$ & $75 \%$ & 50.0 & 10.0 \\
Active Corrective w/ Weight count. & $\mathrm{C}+\mathrm{W}$ & $75 \%$ & 10.0 & 2.0 \\
Active Corrective w/o Weight count. & $\mathrm{C}$ & $0 \%$ & 5.0 & 1.0 \\
Active Weight counterbalance & $\mathrm{W}$ & $75 \%$ & 0.0 & 0.1 \\
Active transparent & $\mathrm{T}$ & $0 \%$ & 0.0 & 0.1 \\
Resistive & $\mathrm{R}$ & $0 \%$ & 0.0 & 3.5 \\
Challenging & $\mathrm{Ch}$ & $-100 \%$ & 0.0 & 0.5 \\
\hline \hline
\end{tabular}

Table 2: The proposed parameters used with the unified compliant controller to render the selected high-level training modalities. $K_{s}$ and $K_{d}$ relate to the impedance-based term. The $W$ parameter corresponds to the weighting factor for the feedforward compensation of Eq. 11.

\subsection{Human-robot interaction modalities results}

We recruited 14 voluntary healthy volunteers, with median age of 25 years [24 - 27]. Table 2 shows the empirically obtained parameters that we used for the human-robot interaction assessment, as described in Section 3.3.

\subsubsection{Kinematics variability assessment}

The results of the trajectory tracking RMSE of the elbow joint are shown in Fig. 10. The overall average tracking error was $3.38 \pm 1.29$ degrees, and the maximum detected RMSE was 5.73 degrees (about 0.1 radians). The Friedman test rejected the null hypothesis that data came from the same distribution ( $\mathrm{p}$-value $<0.0001$ ). The post-hoc analysis revealed that only RMSE data of the Passive mode significantly differed from all the other groups (p-values $<0.01$ ). As expected, since we are using an impedance control logic, which doesn't guarantee an accurate position tracking, and since no effort was required from the user, in Passive mode we can notice higher errors, but the trajectory variability is minimal. Finally, in $W$ mode, by which the controller does not correct for trajectory deviation, the tracking RMSE was slightly higher than the other modes.

\subsubsection{Electromyography results}

In Figure 11, we present the average envelope profiles of muscular contraction (biceps and triceps brachii), and the torque output for each of the presented modality. 


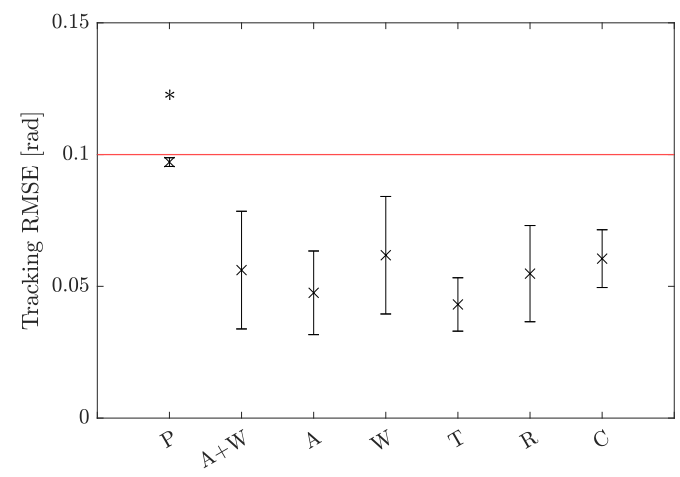

Figure 10: Trajectory tracking RMSE of the elbow joint for each rehabilitation training modality. The red line represents the tolerance threshold of 0.1 radians (about 5.73 degrees) shown in the visual feedback. The asterisk represents significant difference of post-hoc analysis from all the other groups (p-value $<0.05$ with Bonferroni correction). 

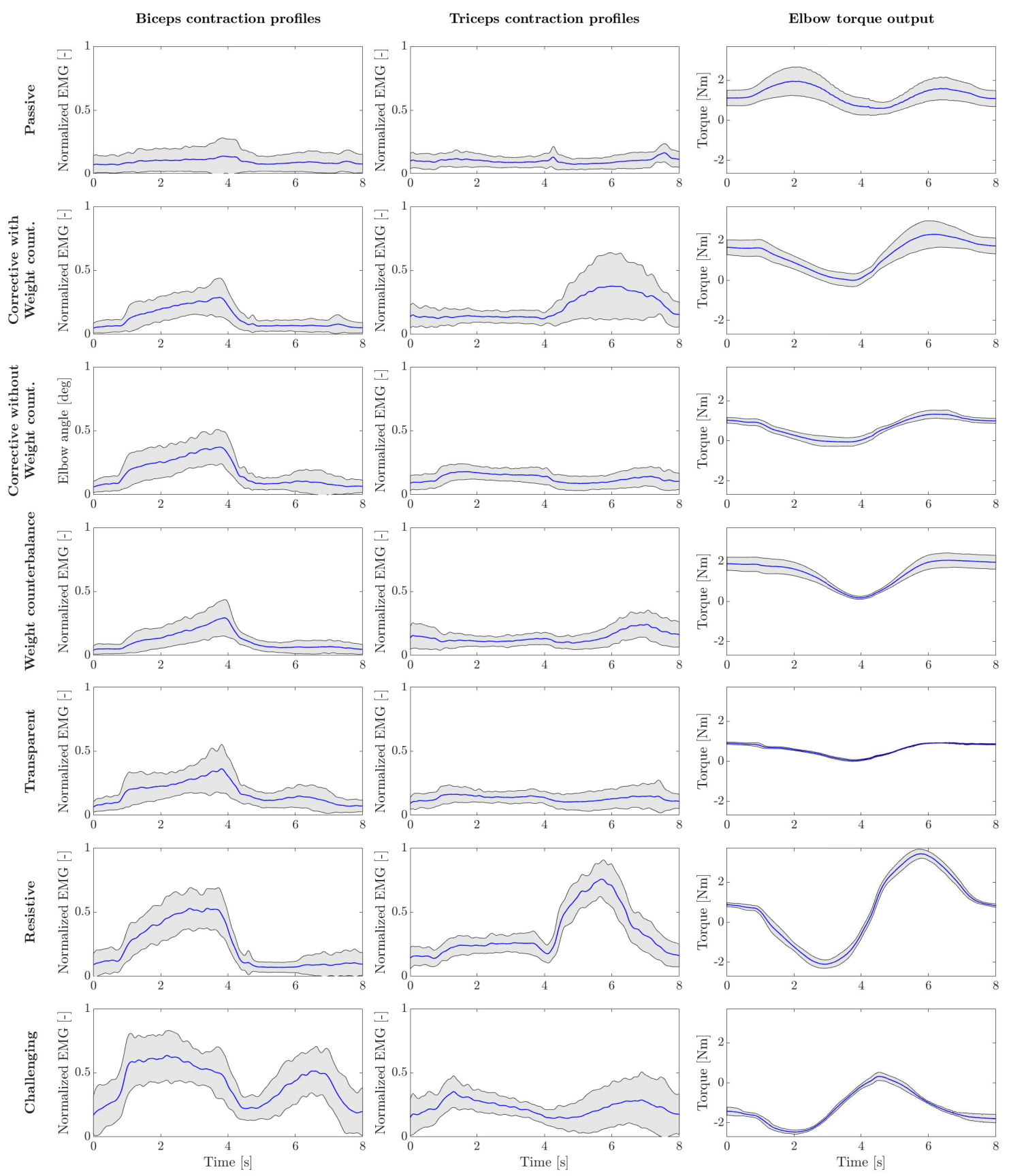

Figure 11: Experimental results for all the presented rehabilitation modalities. Each row represents a different mode. Subplots show biceps and triceps normalized EMG, and measured interaction torque, generated at the output joint axis. Bold blue lines represent mean values, while grey areas refer to standard deviation ranges. 

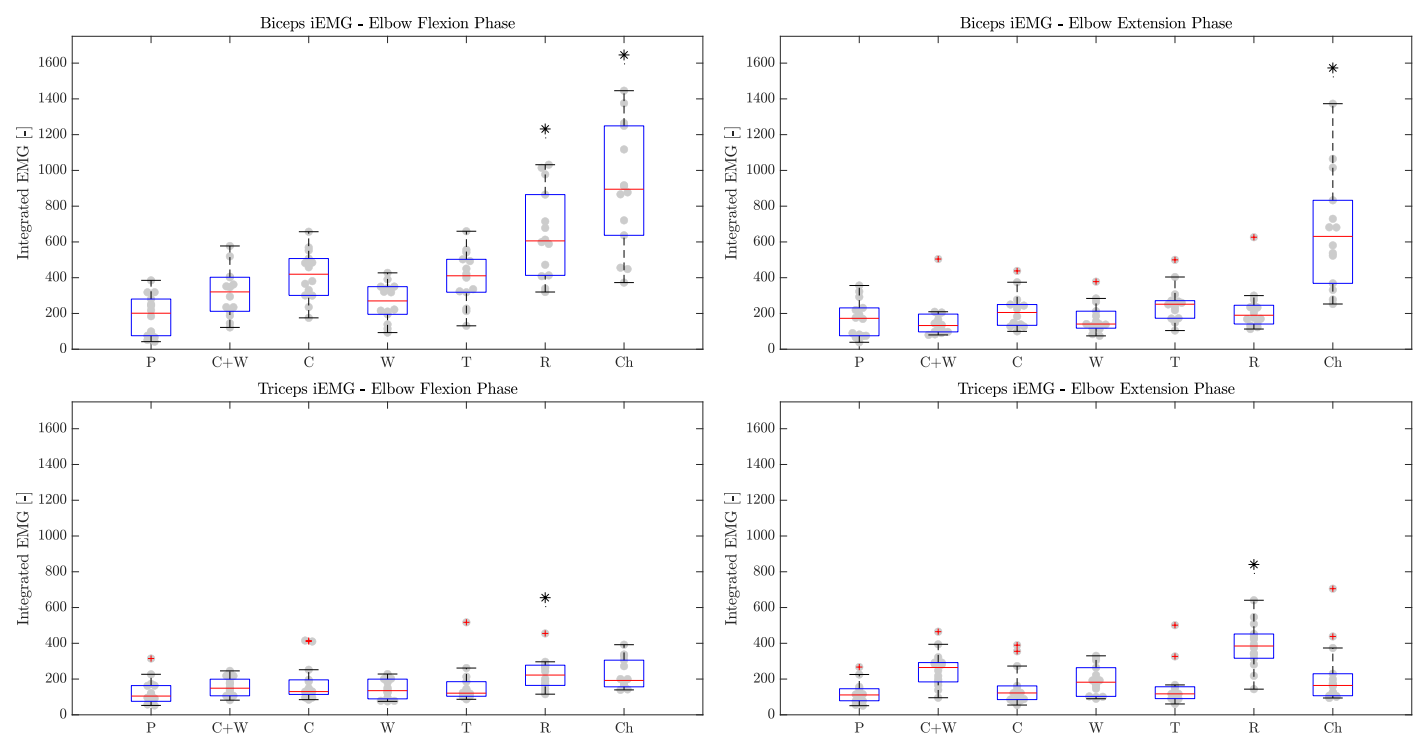

Figure 12: biceps and triceps brachii iEMG during elbow flexion and extension phases. Asterisks indicate those modalities that are significantly different from all the others (p-value $<0.05$ with Bonferroni correction for multiple comparisons). P: Passive mode; $\mathrm{C}+\mathrm{W}$ : Active Corrective with Weight counterbalance; C: Active Corrective without Weight counterbalance; W: Active Weight counterbalance; T: Active Transparent; R: Resistive; C: Challenging

Furthermore, the integrated EMG (iEMG) results are reported in Fig. 12 for each modality. The Friedman test revealed significant differences among training modalities for the iEMG index for the four conditions analyzed (i.e., biceps and triceps contraction during elbow flexion and extension phases) (p-value $<0.0001$ ). Therefore, we performed further analysis to separately compare each rehabilitation modality with the others. The results of the post-hoc analysis are shown in Tables 3 and 4 .

Passive mode (P) In Passive mode, the robot entirely performs the movement and the subjects were asked to simulate the "passive" behavior by relaxing their muscles along the movement, and by not counteracting to residual trajectory errors. As expected, the normalized activation of biceps and triceps was minimal, which confirmed the user's "passive" behavior (Figure 11. Considering the biceps activation during the flexion phase we found a significant difference (i.e., p-values $<0.05$ ) for all modalities except from $W$ mode. Instead, triceps contraction during the extension phase resulted in significant difference with $C+W, W, R$, and $C h$ modes (Table 3 ).

Active Corrective mode with Weight counterbalance $(\mathbf{C}+\mathbf{W})$ Concerning the $C+W$ mode, we can appreciate a distinct activation of the biceps (agonist muscles) during the elbow flexion phase. The biceps activation was not different from $W$ and $T$ modes (p-values $>0.05$ ), while it was significantly different from $P$, where the subjects were almost relaxed, $C$, where the controller did not compensate for gravity, and $R$ and $C h$ modalities, where the subject were making 
more effort (p-values $<0.05)$. In contrast, the triceps (antagonist muscles) contracted during elbow extension. In fact, since the controller was counterbalancing for the arm weight, the users could not exploit the gravity force to extend the elbow during the second phase of the task. Indeed, the iEMG during the triceps extension was significantly higher than the one detected in the $P$ mode where the muscle was relaxed (p-value $<0.001)$ and, at the same time, we did not detect differences from the $W$ mode (p-value $=0.431)$.

Active Corrective mode without Weight counterbalance (C) When in $C$ mode, the activation of the biceps was not statistically different with respect to the $T$ modality ( $p$-value $=0.600$ ), while it was different from the others. The triceps activation plot shows no significant muscular activity during movement in favor of gravity. In fact, the triceps iEMG was not significantly different from the $P$ mode where all the muscles are relaxed (p-value $=0.137$ ). The $C$ mode also demonstrates similarity to the $T$ mode, by which the user substantially uses the contribution gravity in the extension phase, and therefore the triceps activation is almost null.

Active Weight counterbalance mode (W) The trials performed in $W$ mode showed similar muscular activation trends as the $C+W$ mode for both biceps and triceps during the two movement phases. The biceps contracted during the lifting phase, and the triceps during the descending phase. In this mode, since the controller did not correct for trajectory deviation, the trajectory RMSE was higher than the previous modes (Figure 10).

Active Transparent mode (T) Averagely, the users contracted the biceps during the elbow flexion phase and continued to contract during the elbow extension phase to slow down the downward movement. Since the movement was performed against gravity, the triceps muscle was not significantly activated. We can also observe that both biceps and triceps activation profiles of the $T$ mode are substantially similar to $C$ mode (Figure 11). This result is also confirmed also by the iEMG (p-values > 0.05).

Resistive mode (R) In $R$ mode, we can observe high biceps contraction during the elbow flexion phase and triceps contraction during the elbow extension. The activation of the biceps during the elbow flexion was significantly higher than all modalities (p-values $<0.05$ ), except from the $C h$ mode. During the elbow extension phase, we observed triceps contraction significantly greater than all the other training modes, except from the $C h$ one $(\mathrm{p}$-value $=0.066)$.

Challenging mode $(\mathbf{C h})$ The $C h$ mode involved especially the biceps muscles. Indeed, in Figure 11, we can observe a great muscular contraction of the biceps during both elbow flexion and extension. The biceps iEMG index during the elbow flexion phase, was significantly different from all modalities ( $\mathrm{p}$-values $\leq 0.05$ ), except from the $R$ one (p-value $=1.000$ ), where the users were contracting the biceps to overcome the resistance offered by the robot. During the elbow extension, instead, we observed biceps muscular activation significantly higher than all the other training modalities ( $\mathrm{p}$-values $\leq 0.001)$. The triceps, instead, was less active and it was mainly co-contracting. 
Muscular contraction during elbow flexion phase

\begin{tabular}{|c|c|c|c|c|c|c|c|}
\hline Biceps & $\mathbf{P}$ & $\mathbf{C}+\mathbf{W}$ & $\mathrm{C}$ & $\mathbf{W}$ & $\mathbf{T}$ & $\mathbf{R}$ & $\mathbf{C h}$ \\
\hline $\mathbf{P}$ & 1.000 & & & & & & \\
\hline $\mathbf{C}+\mathbf{W}$ & $0.044^{*}$ & 1.000 & & & & & \\
\hline $\mathrm{C}$ & $<0.001^{* *}$ & $0.036^{*}$ & 1.000 & & & & \\
\hline $\bar{W}$ & 0.382 & 0.255 & $0.001^{*}$ & 1.000 & & & \\
\hline $\mathbf{T}$ & $<0.001^{* *}$ & 0.115 & 0.600 & $0.007^{*}$ & 1.000 & & \\
\hline $\mathbf{R}$ & $<0.001^{* *}$ & $<0.001^{* *}$ & $<0.001^{* *}$ & $<0.001 * *$ & $0.036 *$ & 1.000 & \\
\hline $\mathrm{Ch}$ & $<0.001^{* *}$ & $0.004^{*}$ & $<0.001^{* *}$ & $<0.001 * *$ & $<0.001^{* *}$ & 1.000 & 1.000 \\
\hline
\end{tabular}

\begin{tabular}{|c|c|c|c|c|c|c|c|}
\hline Triceps & $\mathbf{P}$ & $\mathrm{C}+\mathrm{W}$ & $\mathrm{C}$ & $\mathbf{W}$ & $\mathbf{T}$ & $\mathbf{R}$ & Ch \\
\hline $\mathbf{P}$ & 1.000 & & & & & & \\
\hline $\mathrm{C}+\mathrm{W}$ & $0.014^{*}$ & 1.000 & & & & & \\
\hline C & $0.002 *$ & 0.499 & 1.000 & & & & \\
\hline W & 0.151 & 0.310 & 0.091 & 1.000 & & & \\
\hline $\mathbf{T}$ & $0.014^{*}$ & 1.000 & 0.499 & 0.310 & 1.000 & & \\
\hline $\mathbf{R}$ & $<0.001 * *$ & $0.002 *$ & $0.014^{*}$ & $<0.001 * *$ & $0.002 *$ & 1.000 & \\
\hline $\mathrm{Ch}$ & $<0.001^{* *}$ & $0.001^{*}$ & $0.011^{*}$ & $<0.001^{* *}$ & $0.001^{*}$ & 0.933 & 1.000 \\
\hline
\end{tabular}

Table 3: P-values results of the post-hoc analysis comparing integrated EMG index among training modalities during elbow flexion movement.

Muscular contraction during elbow extension phase

\begin{tabular}{|c|c|c|c|c|c|c|c|}
\hline Biceps & $\mathbf{P}$ & $\mathrm{C}+\mathrm{W}$ & $\mathrm{C}$ & $\mathbf{W}$ & $\mathbf{T}$ & $\overline{\mathbf{R}}$ & $\mathrm{Ch}$ \\
\hline $\mathbf{P}$ & 1.000 & & & & & & \\
\hline $\mathbf{C}+\mathbf{W}$ & 0.662 & 1.000 & & & & & \\
\hline $\mathrm{C}$ & 0.054 & $0.018^{*}$ & 1.000 & & & & \\
\hline $\mathbf{W}$ & 0.726 & 0.431 & 0.115 & 1.000 & & & \\
\hline $\mathbf{T}$ & $0.004^{*}$ & $<0.001^{* *}$ & 0.336 & $0.011^{*}$ & 1.000 & & \\
\hline $\mathbf{R}$ & $0.036^{*}$ & $0.011^{*}$ & 0.861 & 0.080 & 0.431 & 1.000 & \\
\hline $\mathrm{Ch}$ & $<0.001^{* *}$ & $<0.001^{* *}$ & $<0.001^{* *}$ & $<0.001^{* *}$ & $<0.001^{* *}$ & $<0.001^{* *}$ & 1.000 \\
\hline
\end{tabular}

\begin{tabular}{|c|c|c|c|c|c|c|c|}
\hline Triceps & $\mathbf{P}$ & $\mathbf{C}+\mathbf{W}$ & $\mathrm{C}$ & $\mathbf{W}$ & $\mathbf{T}$ & $\mathbf{R}$ & Ch \\
\hline $\mathbf{P}$ & 1.000 & & & & & & \\
\hline $\mathbf{C}+\mathbf{W}$ & $<0.001^{* *}$ & 1.000 & & & & & \\
\hline $\mathrm{C}$ & 0.137 & $0.029 *$ & 1.000 & & & & \\
\hline $\mathbf{W}$ & $0.004^{*}$ & 0.431 & 0.162 & 1.000 & & & \\
\hline $\bar{T}$ & 0.096 & $0.044^{*}$ & 0.861 & 0.221 & 1.000 & & \\
\hline $\mathbf{R}$ & $<0.001^{* *}$ & $0.029 *$ & $<0.001^{* *}$ & $0.003^{*}$ & $0.018^{*}$ & 1.000 & \\
\hline $\mathrm{Ch}$ & $<0.001^{* *}$ & 0.726 & $0.011^{*}$ & 0.255 & $<0.001^{* *}$ & 0.066 & 1.000 \\
\hline
\end{tabular}

Table 4: P-values results of the post-hoc analysis comparing integrated EMG index among training modalities during elbow extension movement. 


\subsubsection{Torque output results}

Regarding the torque output results presented in Fig. 11, the right plots show the torque output generated by the elbow-joint system to the users' arm interface. In $P$ mode, the measured torque consisted of the torque generated by the motor to complete the task. Such torque is equal to the inverse-dynamic torque needed to passively move the human-robot system along the desired trajectory, besides residual torques that are not rejected by the torque controller. In $C+W$ mode the torque output is composed of both the impedance-based and the counterbalance torques, while in $C$ mode only the impedance-based torque is present. For this reason, in $C+W$ mode we can notice a greater variability, which is introduced by the feedforward term that depends on the users' arm weight. In $W$ mode, the system compensates for arm weight, which varies according to the wearer characteristics. This explains the greater variability and the greater amplitude of torque profiles. In $T$ mode, instead, the robot only compensates its weight, with no trajectory correction. Accordingly, the measured torque profiles show a smaller variance, and the trend goes deterministically with the cosine of the joint position, as described in Eq. 10. The $R$ mode shows that torque trends are inversely proportional to the task velocity, demonstrating a viscous frictional behavior. Finally, a visual inspection shows that in $C h$ mode, the torque output was opposite to the $P$ mode. In fact, the assistance in $P$ mode was pushing the arm in the opposite direction with respect to the $C h$ mode, in which the torque output is aligned with the gravity direction.

\section{Discussion}

The literature proposed several high-level training modalities for effective post-stroke rehabilitation treatment. However, their implementation strongly depends on the developed robotic systems. For example, the Harmony exoskeleton exploits an explicit SEA-based impedance controller [39], which is similar to our approach, while other exoskeletons, such as ARMin, use instead implicit impedance controllers to promote rehabilitation exercises $[36,53]$. However, the generalization of these approaches to a large variety of human-robot interaction rehabilitation modalities, and their integration in a unified low-level compliant controller, have not been investigated yet.

This work aimed to design and develop a unified control framework for upper-limb robotmediated rehabilitation that could implement different high-level rehabilitation modalities under a unified low-level control law and to evaluate the muscular engagement of the multiple control solutions.

\subsection{Actuation and control}

As a first step, we identified a suitable actuation configuration that could be exploited to create a compliant joint for upper-limb rehabilitation robots. We used actuators along with load-cell feedback to provide high-fidelity torque control. In this way, low-impedance behavior can be achieved, and the robot can behave compliantly with respect to the subject, encouraging residual voluntary movements. On top of this configuration, we proposed a generalized explicit impedance-based control law, which includes positive-feedback terms for friction compensation and arm weight counterbalance. We tested the unified controller performances with an elbow flexion-extension test-bed. The experimental results showed that the developed set-up, combined with the proposed low-level controller, exhibited very low impedance at the joint level, imposing negligible resistive torques (less than $0.3 \mathrm{Nm}$ ) on the user's free-motion movements. Notably, since the impedance-based corrective 
term of the unified controller is superimposed to the Transparent control mode, achieving a baseline dynamic transparent behavior was a fundamental step to implement compliant rehabilitation strategies. We can conclude that the inner-loop is expected not to influence the high-level behavior and it can be considered an ideal torque source. With these results, we demonstrated that the proposed approach was effective in implementing different virtual stiffness and damping values, that were performed by the robot with good accuracy. Coupled stability and passivity considerations presented in Section 3.4 suggest that a more in-depth analysis should be performed to guarantee the stability of the human-robot system.

\subsection{Human-robot interaction modalities}

With the developed system, we proposed a set of parameters that could implement different physical human-robot behavior. Specifically, we combined assistance, correction and resistance to promote a collaborative controller that implements different high-level training modalities. All the previously presented discrete robot-mediated training strategies can be viewed as different points of a continuum of corrective assistance, counterbalance assistance, and resistance. We underline that the aim of this work is not to define a single set of parameters, but to test the hypothesis that the parameter space, if properly explored, can be exploited to move across different rehabilitation scenarios. In particular, we included and tested seven rehabilitation modalities, as described in Section 3.3.

In this work, we evaluated the capability of the proposed framework to promote different training modalities levels by measuring the voluntary muscular activity in a controlled experimental protocol. We compared biceps and triceps muscular activity of 14 healthy subjects under the identified rehabilitation modalities. At the same time, the angular position followed by users and the torque output generated by the elbow-joint system were measured.

The kinematics experimental results clearly demonstrated that the subjects could keep the full control of the robotic link while performing elbow flexion/extension tasks. Consequently, the results confirm two crucial hypotheses. Firstly, participants' kinematics performances did not show significant difference across the presented training modalities. Secondly, all the subjects were able to follow the desired trajectory within the maximum tolerance of about 0.1 radians (about 5.73 degrees). For these reasons, we posit that, under all tested conditions, all subjects could fulfill the required motor tasks in terms of trajectory tracking, range of movement and timing, no matter the level of assistance/resistance provided. Thus, we could compare the electromyographic data across modalities. We observed trajectory tracking to be less accurate than in a position controlled system (especially for the $P$ mode). In fact, the impedance control scheme, due to the pure springdamper correction, introduces bias offset errors to the trajectory tracking control problem that are not negligible. Contrarily, a position control scheme would reject such errors, but it would not provide compliant behavior with the human arm. Furthermore, in applications by which the robot is coupled with a fragile human arm, achieving precise positioning is not a critical aspect, but it is more important to avoid high interaction torques that can be uncomfortable or potentially hazardous to the wearer.

As desired, we observed that the different human-robot interaction modalities implemented with the unified controller induced different muscular activation patterns, both in biceps and triceps brachii, according to the selected training modality. The interaction modalities ranged from a full robot action with almost null muscular contribution (Passive $(P)$ mode), to training paradigms where the robot resists and challenges the users, requiring them an extra muscular effort to accomplish the task (Resistive ( $R$ ) and Challenging ( $C h$ ) modes). 
The Transparent (T) mode was considered the baseline reference, since it describes the behavior by which neither assistance nor resistance is provided to the user during the task. In fact, the muscular effort registered in this modality corresponds to the natural free task execution. During elbow-flexion we observed a medium biceps contraction, while the triceps was characterized by a slight co-contraction. During the extension phase, instead, a modulated contraction of the biceps is used to control the downward motion provided by gravity, while the triceps were again not significantly activated, given that the movement was performed in favour of gravity.

We also observed that Assistive $(C, W$ and $C+W)$ modes promoted similar biceps contractions that are significantly higher with respect to Passive mode. However, when the weight counterbalance was active (i.e, $C+W$ and $W$ modalities), the triceps experienced greater contraction with respect to the other training modes. Therefore, these results indicate that such modalities induced the physiological contraction of biceps muscles, and that the controller was inducing slightly greater motor antagonistic activation when additional weight counterbalance assistance was present. Comparing results obtained in $T$ mode with the $C$ mode, we could interestingly observe that the activation profiles in the two modalities were comparable, despite the $C$ mode allows a reduced effort and avoid any fail in task execution, providing assistance whether the user is not capable of completing the task or is too slow. We can also observe that, given that the participants were performing controlled movements (i.e., healthy subjects followed a trajectory pre-defined in position and velocity) with comparable performances, the controller was able to induce muscular patterns in the $A, A+W$ and $W$ modes that are not significantly different from the baseline $T$ mode. We can also verify that the torque output in this modalities roughly followed the robot weight counterbalance term, and that the residual dynamic torque to complete the tasks was generated by users' voluntary contraction. Therefore, we can derive that the proposed control system is able to correctly implement the assist-as-needed paradigm, helping the user to accomplish the task while inducing the physiological muscular activation pattern.

Instead, in $R$ and $C h$ modes, the statistical analysis confirmed that, for both biceps and triceps, significant greater muscular contraction levels were reached with respect to other modalities. In particular, the $C h$ mode can be regarded as equivalent to gym-like exercises. In fact, the robot trained the biceps along the whole movement, during both elbow flexion and extension movements, as if the user was performing the task with payload weights. On the contrary, in $R$ mode the robot trained both muscles during the task: the biceps contracted during the flexion phase, and the triceps during the extension phase.

The results demonstrated that the proposed unified controller was able to provide low-impedance and high-impedance correction, low-resistance and high-resistance behavior, rendering different perceived human-robot interaction modalities. The developed controller, thanks to its inner explicit torque feedback control, could reject most of the disturbance torques introduced by the high-ratio gearbox, without the need for an accurate model-based compensation.

From the rehabilitation point of view, the goal is to achieve efficient motor control that should be as similar as possible to the free task scenario, i.e., the Transparent mode. However, we noticed that $W$ and $C+W$ solutions, which both involved anti-gravity compensation, imply an agonistantagonist coordination that is completely different from the natural one, and therefore they could induce unnatural muscular synergies. Instead, purely corrective strategies (such as $C$ mode), around the desired trajectory, modulate the assistance without impacting the muscle recruitment strategy, but guaranteeing the completion of the task. Contrarily to what historically researchers tended to do, we suggest not to exploit purely counterbalancing strategies for post-stroke rehabilitation, since lightening the human arm could induce different motor control learning with respect to corrective 
strategies.

Instead, the proposed $R$ and $C h$ methods were able to motivate and induce challenging exercises to the subject, training both agonist and antagonist muscles. For this reason the presented approach could also be applied to the recovery from sports and non-sports injuries. In fact, the controller might assist the motion during early stages of the physiotherapy, then, by switching modality, it might improve the muscle mass recovery.

Overall, the controller and the developed hardware confirmed suitability to implement the training modalities needed for an effective physical-therapy treatment. With these advancements, we can conclude that the proposed compliant controller might assist the patient along the upper-limb rehabilitation treatment process, from stages when the patient is completely hemiplegic towards the functional recovery of the limb.

Although in this work we developed a compliant joint for the elbow training, future works can involve the translation of the proposed solution to multi degree-of-freedom applications. Indeed, the joint-space control scheme can be replicated for each joint of the robotic chain, and more sophisticated centralized algorithms for arm weight compensation can be implemented.

\section{Conclusion}

In this paper, we presented and tested a human-robot cooperative controller for upper-limb robotmediated rehabilitation. The design of the control framework took inspiration from motor learning and neurophysiological aspects, which suggest that good collaboration between the impaired subject and the therapeutic device is needed to induce an effective motor recovery. In this sense, we found strong evidence that the proposed controller guaranteed dynamic transparency - to promote users' voluntary movements - and produced variable assistance and resistance levels - to tune the rehabilitation treatment according to subject's performance and involvement.

We demonstrated that a proper combination of stiffness, damping, and weight assistance of the presented unified controller can render different physical human-robot interaction and, consequently, promote different human-robot interaction rehabilitation modalities. We also proved that assistance based on anti-gravity weight counterbalance (i.e., $W$ and $C+W$ modes) changes the muscular effort with respect to purely corrective assistance (i.e. $C$ mode). Thus it does not train the same muscular synergic coordination of natural free task movements. We believe that, since a collaborative controller should provide the minimal amount of assistance to complete the tasks, the presented high-level modalities can be considered as different points of a continuum, and we posit that they can be potentially selectable according to the stage of motor recovery, involving the subject in the completion of the rehabilitation treatment. Our results suggest that the presented collaborative framework is suitable for these purposes. Future works will extend this approach to multiple degrees of freedom robots and investigate the optimal adaptation control law that makes the controller learn and adapt to the subject's performances in a therapist-like manner.

\section{Declarations}

\section{Ethics approval and consent to participate}

This study was submitted and approved by the institutional Ethical Commitee of Politecnico di Milano. 


\section{Consent for publication}

Written informed consent was obtained from all human subjects for the publication of this report and any accompanying images

\section{Availability of data and materials}

The dataset used and/or analysed during the current study are available from the corresponding author upon reasonable request.

\section{Competing interests}

SDG, FB, AP and MG have interests in AGADE Srl, Milano, Italy.

\section{Funding}

This work has been performed thanks to the AGREE project, and it was partially supported by Regione Lombardia, Italy (Tender ARIA (formerly ARCA) 2018_132).

\section{Authors' contributions}

SDG, MG, AP, FB conceived the presented idea. SDG developed the theory and implemented control architecture. SDG, MG, VL conceived testing protocols. SDG, VL performed measurements. SDG, VL, MG performed data analysis. SDG, VL drafted the manuscript. All authors discussed the results and contributed to the final manuscript. All authors made a significant contribution to the review of the manuscript, read and approved the final manuscript.

\section{Acknowledgements}

We thank the volunteers who participated to the study.

\section{Author details}

${ }^{1}$ NeuroEngineering And medical Robotics Laboratory (NearLab), Department of Electronics, Information and Bioengineering, Politecnico di Milano, Milano, Italy.

${ }^{2}$ Department of Mechanical Engineering, Politecnico di Milano, Milano, Italy. Correspondence to stefano.dallagasperina@polimi.it

\section{References}

[1] Alin Albu-Schäffer, Christian Ott, and Gerd Hirzinger. A unified passivity-based control framework for position, torque and impedance control of flexible joint robots. The international journal of robotics research, 26(1):23-39, 2007.

[2] Emilia Ambrosini, Stefano Dalla Gasperina, Marta Gandolla, and Alessandra Pedrocchi. Upper-limb exoskeletons for stroke rehabilitation. In Mediterranean Conference on Medical and Biological Engineering and Computing, pages 1722-1728. Springer, 2019. 
[3] Ghaith J Androwis, Rakesh Pilkar, Arvind Ramanujam, and Karen J Nolan. Electromyography assessment during gait in a robotic exoskeleton for acute stroke. Frontiers in neurology, 9:630, 2018.

[4] B Armstrong and C de Wit. Canudas," friction modeling and compensation", the control handbook, 1995.

[5] Angelo Basteris, Sharon M. Nijenhuis, Arno H.A. Stienen, Jaap H. Buurke, Gerdienke B. Prange, and Farshid Amirabdollahian. Training modalities in robot-mediated upper limb rehabilitation in stroke: A framework for classification based on a systematic review, jul 2014.

[6] Thiago Boaventura, Gustavo A Medrano-Cerda, Claudio Semini, Jonas Buchli, and Darwin G Caldwell. Stability and performance of the compliance controller of the quadruped robot hyq. In 2013 IEEE/RSJ International Conference on Intelligent Robots and Systems, pages 1458 1464. IEEE, 2013.

[7] Thiago Boaventura, Claudio Semini, Jonas Buchli, Marco Frigerio, Michele Focchi, and Darwin G Caldwell. Dynamic torque control of a hydraulic quadruped robot. In 2012 IEEE international conference on robotics and automation, pages 1889-1894. IEEE, 2012.

[8] Andrea Calanca, Riccardo Muradore, and Paolo Fiorini. A review of algorithms for compliant control of stiff and fixed-compliance robots. IEEE/ASME Transactions on Mechatronics, $21(2): 613-624,2016$.

[9] Andrea Calanca, Riccardo Muradore, and Paolo Fiorini. Impedance control of series elastic actuators: Passivity and acceleration-based control. Mechatronics, 47:37-48, 2017.

[10] Jim D Chapel and Renjeng Su. Coupled stability characteristics of nearly passive robots. In Proceedings 1992 IEEE International Conference on Robotics and Automation, pages 1342 1343. IEEE Computer Society, 1992.

[11] Tianyao Chen, Rafael Casas, and Peter S Lum. An elbow exoskeleton for upper limb rehabilitation with series elastic actuator and cable-driven differential. IEEE Transactions on Robotics, 35(6):1464-1474, 2019.

[12] J Edward Colgate and J Michael Brown. Factors affecting the z-width of a haptic display. In Proceedings of the 1994 IEEE International Conference on Robotics and Automation, pages 3205-3210. IEEE, 1994.

[13] James Edward Colgate and Neville Hogan. Robust control of dynamically interacting systems. International journal of Control, 48(1):65-88, 1988.

[14] John J Craig. Introduction to robotics: mechanics and control, 3/E. Pearson Education India, 2009.

[15] Simona Crea, Marco Cempini, Matteo Moise, Alessia Baldoni, Emilio Trigili, D Marconi, Mario Cortese, Francesco Giovacchini, Federico Posteraro, and Nicola Vitiello. A novel shoulderelbow exoskeleton with series elastic actuators. In 2016 6th IEEE International Conference on Biomedical Robotics and Biomechatronics (BioRob), pages 1248-1253. IEEE, 2016. 
[16] Ana C de Oliveira, Kevin Warburton, James S Sulzer, and Ashish D Deshpande. Effort estimation in robot-aided training with a neural network. In 2019 International Conference on Robotics and Automation (ICRA), pages 563-569. IEEE, 2019.

[17] Christophe Duret, Anne Gaëlle Grosmaire, and Hermano Igo Krebs. Robot-assisted therapy in upper extremity hemiparesis: Overview of an evidence-based approach, apr 2019.

[18] Michele Focchi, Gustavo A Medrano-Cerda, Thiago Boaventura, Marco Frigerio, Claudio Semini, Jonas Buchli, and Darwin G Caldwell. Robot impedance control and passivity analysis with inner torque and velocity feedback loops. Control Theory and Technology, 14(2):97-112, 2016.

[19] Beverley French, Lois H. Thomas, Jacqueline Coupe, Naoimh E. Mcmahon, Louise Connell, Joanna Harrison, Christopher J. Sutton, Svetlana Tishkovskaya, and Caroline L. Watkins. Repetitive task training for improving functional ability after stroke, nov 2016.

[20] Antonio Frisoli, Fabio Salsedo, Massimo Bergamasco, Bruno Rossi, and Maria C Carboncini. A force-feedback exoskeleton for upper-limb rehabilitation in virtual reality. Applied Bionics and Biomechanics, 6(2):115-126, 2009.

[21] Marta Gandolla, Eleonora Guanziroli, Andrea D'Angelo, Giovanni Cannaviello, Franco Molteni, and Alessandra Pedrocchi. Automatic setting procedure for exoskeleton-assisted overground gait: proof of concept on stroke population. Frontiers in neurorobotics, 12:10, 2018.

[22] Philip B Gorelick. The global burden of stroke: persistent and disabling. The Lancet Neurology, 18(5):417-418, 2019.

[23] Marco Guidali, Alexander Duschau-Wicke, Simon Broggi, Verena Klamroth-Marganska, Tobias Nef, and Robert Riener. A robotic system to train activities of daily living in a virtual environment. Medical \& biological engineering \& computing, 49(10):1213-1223, 2011.

[24] Muhammad Ahsan Gull, Shaoping Bai, and Thomas Bak. A review on design of upper limb exoskeletons. Robotics, 9(1):16, 2020.

[25] Takeshi Hatanaka, Nikhil Chopra, and Mark W Spong. Passivity-based control of robots: Historical perspective and contemporary issues. In 2015 44th IEEE Conference on Decision and Control (CDC), pages 2450-2452. IEEE, 2015.

[26] Neville Hogan. An organizing principle for a class of voluntary movements. Journal of Neuroscience, 4(11):2745-2754, 1984.

[27] Neville Hogan. Impedance control: An approach to manipulation: Part i-theory. 1985.

[28] Neville Hogan. Controlling impedance at the man/machine interface. In 1989 IEEE International Conference on Robotics and automation, pages 1626-1627. IEEE Computer Society, 1989.

[29] Vincent S Huang and John W Krakauer. Robotic neurorehabilitation: a computational motor learning perspective. Journal of neuroengineering and rehabilitation, 6(1):1-13, 2009. 
[30] Riccardo Iandolo, Francesca Marini, Marianna Semprini, Matteo Laffranchi, Maddalena Mugnosso, Amel Cherif, Lorenzo De Michieli, Michela Chiappalone, and Jacopo Zenzeri. Perspectives and challenges in robotic neurorehabilitation. Applied Sciences, 9(15):3183, 2019.

[31] Walter Johnson, Oyere Onuma, Mayowa Owolabi, and Sonal Sachdev. Stroke: a global response is needed. Bulletin of the World Health Organization, 94(9):634, 2016.

[32] Fabian Just, Özhan Özen, Philipp Bösch, Hanna Bobrovsky, Verena Klamroth-Marganska, Robert Riener, and Georg Rauter. Exoskeleton transparency: Feed-forward compensation vs. disturbance observer. At-Automatisierungstechnik, 66(12):1014-1026, 2018.

[33] Fabian Just, Özhan Özen, Stefano Tortora, Verena Klamroth-Marganska, Robert Riener, and Georg Rauter. Human arm weight compensation in rehabilitation robotics: Efficacy of three distinct methods. Journal of NeuroEngineering and Rehabilitation, 17(1):1-17, 2020.

[34] Fabian Just, Özhan Özen, Stefano Tortora, Robert Riener, and Georg Rauter. Feedforward model based arm weight compensation with the rehabilitation robot armin. In 2017 International Conference on Rehabilitation Robotics (ICORR), pages 72-77. IEEE, 2017.

[35] Arvid QL Keemink, Herman van der Kooij, and Arno HA Stienen. Admittance control for physical human-robot interaction. The International Journal of Robotics Research, 37(11):1421$1444,2018$.

[36] Abdul Manan Khan, Deok-won Yun, Mian Ashfaq Ali, Jungsoo Han, Kyoosik Shin, and Changsoo Han. Adaptive impedance control for upper limb assist exoskeleton. In 2015 IEEE International Conference on Robotics and Automation (ICRA), pages 4359-4366. IEEE, 2015.

[37] Bongsu Kim and Ashish D Deshpande. Controls for the shoulder mechanism of an upper-body exoskeleton for promoting scapulohumeral rhythm. In 2015 IEEE International Conference on Rehabilitation Robotics (ICORR), pages 538-542. IEEE, 2015.

[38] Bongsu Kim, Aurelien Rodot, and Ashish D Deshpande. Impedance control based on a position sensor in a rehabilitation robot. In ASME 2014 Dynamic Systems and Control Conference. American Society of Mechanical Engineers Digital Collection.

[39] Gayeong Kim, Seung Yeop Lim, Hyun Jong Kim, Byung Joon Lee, Seung Chul Seo, Ki Hun Cho, and Wan Hee Lee. Is robot-assisted therapy effective in upper extremity recovery in early stage stroke? - a systematic literature review, 2017.

[40] Kyoungchul Kong, Hyosang Moon, Doyoung Jeon, and Masayoshi Tomizuka. Control of an exoskeleton for realization of aquatic therapy effects. IEEE/ASME Transactions on Mechatronics, 15(2):191-200, 2010.

[41] John W Krakauer. Motor learning: its relevance to stroke recovery and neurorehabilitation. Current opinion in neurology, 19(1):84-90, 2006.

[42] Hermano Igo Krebs, Mindy L Aisen, Bruce T Volpe, and Neville Hogan. Quantization of continuous arm movements in humans with brain injury. Proceedings of the National Academy of Sciences, 96(8):4645-4649, 1999. 
[43] Hermano Igo Krebs, Jerome Joseph Palazzolo, Laura Dipietro, Mark Ferraro, Jennifer Krol, Keren Rannekleiv, Bruce T Volpe, and Neville Hogan. Rehabilitation robotics: Performancebased progressive robot-assisted therapy. Autonomous robots, 15(1):7-20, 2003.

[44] Ying Mao and Sunil Kumar Agrawal. Design of a cable-driven arm exoskeleton (carex) for neural rehabilitation. IEEE Transactions on Robotics, 28(4):922-931, 2012.

[45] Laura Marchal-Crespo and David J Reinkensmeyer. Review of control strategies for robotic movement training after neurologic injury. Journal of neuroengineering and rehabilitation, $6(1): 1-15,2009$.

[46] Stefano Masiero, Andrea Celia, Giulio Rosati, and Mario Armani. Robotic-Assisted Rehabilitation of the Upper Limb After Acute Stroke. Archives of Physical Medicine and Rehabilitation, 88(2):142-149, 22007.

[47] Nauman Masud, Christian Smith, and Magnus Isaksson. Disturbance observer based dynamic load torque compensator for assistive exoskeletons. Mechatronics, 54:78-93, 2018.

[48] Haifa Mehdi and Olfa Boubaker. Stiffness and impedance control using lyapunov theory for robot-aided rehabilitation. International Journal of Social Robotics, 4(1):107-119, 2012.

[49] Jan Mehrholz, Marcus Pohl, Thomas Platz, Joachim Kugler, and Bernhard Elsner. Electromechanical and robot-assisted arm training for improving activities of daily living, arm function, and arm muscle strength after stroke. Cochrane Database of Systematic Reviews, (9), 2018.

[50] Jan Mehrholz, Alex Pollock, Marcus Pohl, Joachim Kugler, and Bernhard Elsner. Systematic review with network meta-analysis of randomized controlled trials of robotic-assisted arm training for improving activities of daily living and upper limb function after stroke. Journal of neuroengineering and rehabilitation, 17(1):1-14, 2020.

[51] Wei Meng, Quan Liu, Zude Zhou, Qingsong Ai, Bo Sheng, and Shengquan Shane Xie. Recent development of mechanisms and control strategies for robot-assisted lower limb rehabilitation. Mechatronics, 31:132-145, 2015.

[52] Mavuto M Mukaka. A guide to appropriate use of correlation coefficient in medical research. Malawi medical journal, 24(3):69-71, 2012.

[53] Tobias Nef, Matjaz Mihelj, Gabriela Kiefer, Christina Perndl, Roland Muller, and Robert Riener. Armin-exoskeleton for arm therapy in stroke patients. In 2007 IEEE 10th international conference on rehabilitation robotics, pages 68-74. IEEE, 2007.

[54] Tobias Nef, Matjaz Mihelj, and Robert Riener. Armin: a robot for patient-cooperative arm therapy. Medical \&3 biological engineering \& computing, 45(9):887-900, 2007.

[55] Ali Utku Pehlivan, Dylan P. Losey, and Marcia K. Omalley. Minimal Assist-as-Needed Controller for Upper Limb Robotic Rehabilitation. IEEE Transactions on Robotics, 32(1):113-124, feb 2016.

[56] Ali Utku Pehlivan, Chad Rose, and Marcia K O'Malley. System characterization of ricewrist-s: a forearm-wrist exoskeleton for upper extremity rehabilitation. In 2013 IEEE 13th International Conference on Rehabilitation Robotics (ICORR), pages 1-6. IEEE, 2013. 
[57] Juan C. Perez-Ibarra, Adriano A.G. Siqueira, and Hermano I. Krebs. Assist-As-needed ankle rehabilitation based on adaptive impedance control. IEEE International Conference on Rehabilitation Robotics, 2015-Septe:723-728, 2015.

[58] Tommaso Proietti, Vincent Crocher, Agnes Roby-Brami, and Nathanael Jarrasse. Upper-Limb Robotic Exoskeletons for Neurorehabilitation: A Review on Control Strategies. IEEE Reviews in Biomedical Engineering, 9:4-14, 2016.

[59] Tommaso Proietti, Nathanaël Jarrassé, Agnès Roby-Brami, and Guillaume Morel. Adaptive control of a robotic exoskeleton for neurorehabilitation. In 2015 7th International IEEE/EMBS Conference on Neural Engineering (NER), pages 803-806. IEEE, 2015.

[60] David J Reinkensmeyer, Etienne Burdet, Maura Casadio, John W Krakauer, Gert Kwakkel, Catherine E Lang, Stephan P Swinnen, Nick S Ward, and Nicolas Schweighofer. Computational neurorehabilitation: modeling plasticity and learning to predict recovery. Journal of neuroengineering and rehabilitation, 13(1):1-25, 2016.

[61] Anthony L Ricamato and Joseph M Hidler. Quantification of the dynamic properties of emg patterns during gait. Journal of electromyography and kinesiology, 15(4):384-392, 2005.

[62] Andres F Ruiz, Eduardo Rocon, and Arturo Forner-Cordero. Exoskeleton-based robotic platform applied in biomechanical modelling of the human upper limb. Applied Bionics and Biomechanics, 6(2):205-216, 2009.

[63] Marie Schumacher, Janis Wojtusch, Philipp Beckerle, and Oskar von Stryk. An introductory review of active compliant control. Robotics and Autonomous Systems, 119:185-200, 2019.

[64] Bruno Siciliano, Lorenzo Sciavicco, Luigi Villani, and Giuseppe Oriolo. Robotics: modelling, planning and control. Springer Science \& Business Media, 2010.

[65] Zhibin Song, Shuxiang Guo, Muye Pang, Songyuan Zhang, Nan Xiao, Baofeng Gao, and Liwei Shi. Implementation of resistance training using an upper-limb exoskeleton rehabilitation device for elbow joint. J. Med. Biol. Eng, 34(2):188-196, 2014.

[66] Heike Vallery, Jan Veneman, Edwin Van Asseldonk, Ralf Ekkelenkamp, Martin Buss, and Herman Van Der Kooij. Compliant actuation of rehabilitation robots. IEEE Robotics 8 Automation Magazine, 15(3):60-69, 2008.

[67] Janne M Veerbeek, Anneli C Langbroek-Amersfoort, Erwin EH Van Wegen, Carel GM Meskers, and Gert Kwakkel. Effects of robot-assisted therapy for the upper limb after stroke: a systematic review and meta-analysis. Neurorehabilitation and neural repair, 31(2):107-121, 2017.

[68] Carolee J. Winstein, Joel Stein, Ross Arena, Barbara Bates, Leora R. Cherney, Steven C. Cramer, Frank Deruyter, Janice J. Eng, Beth Fisher, Richard L. Harvey, Catherine E. Lang, Marilyn MacKay-Lyons, Kenneth J. Ottenbacher, Sue Pugh, Mathew J. Reeves, Lorie G. Richards, William Stiers, and Richard D. Zorowitz. Guidelines for Adult Stroke Rehabilitation and Recovery: A Guideline for Healthcare Professionals from the American Heart Association/American Stroke Association, jun 2016.

[69] David A Winter. Biomechanics and motor control of human movement. John Wiley \& Sons, 2009. 
[70] Eric T Wolbrecht, Vicky Chan, David J Reinkensmeyer, and James E Bobrow. Optimizing compliant, model-based robotic assistance to promote neurorehabilitation. IEEE Transactions on Neural Systems and Rehabilitation Engineering, 16(3):286-297, 2008.

[71] Kuan-Yi Wu, Yin-Yu Su, Ying-Lung Yu, Ching-Hui Lin, and Chao-Chieh Lan. A 5-Degreesof-Freedom Lightweight Elbow-Wrist Exoskeleton for Forearm Fine-Motion Rehabilitation. IEEE/ASME Transactions on Mechatronics, 24(6):2684-2695, dec 2019.

[72] Damiano Zanotto, Tommaso Lenzi, Paul Stegall, and Sunil K Agrawal. Improving transparency of powered exoskeletons using force/torque sensors on the supporting cuffs. In 2013 IEEE 13th International Conference on Rehabilitation Robotics (ICORR), pages 1-6. IEEE, 2013. 